\title{
Relative cue encoding in the context of sophisticated models of categorization: Separating information from categorization
}

\author{
Keith S. Apfelbaum • Bob McMurray
}

Published online: 5 December 2014

(C) Psychonomic Society, Inc. 2014

\begin{abstract}
Traditional studies of human categorization often treat the processes of encoding features and cues as peripheral to the question of how stimuli are categorized. However, in domains where the features and cues are less transparent, how information is encoded prior to categorization may constrain our understanding of the architecture of categorization. This is particularly true in speech perception, where acoustic cues to phonological categories are ambiguous and influenced by multiple factors. Here, it is crucial to consider the joint contributions of the information in the input and the categorization architecture. We contrasted accounts that argue for raw acoustic information encoding with accounts that posit that cues are encoded relative to expectations, and investigated how two categorization architectures - exemplar models and back-propagation parallel distributed processing modelsdeal with each kind of information. Relative encoding, akin to predictive coding, is a form of noise reduction, so it can be expected to improve model accuracy; however, like predictive coding, the use of relative encoding in speech perception by humans is controversial, so results are compared to patterns of human performance, rather than on the basis of overall accuracy. We found that, for both classes of models, in the vast majority of parameter settings, relative cues greatly helped the models approximate human performance. This suggests that expectation-relative processing is a crucial precursor step in phoneme categorization, and that understanding the information content is essential to understanding categorization processes.
\end{abstract}

K. S. Apfelbaum $(\bowtie)$

Department of Psychology, Ohio State University, Psychology

Building, 1835 Neil Ave, Columbus, OH 43210, USA

e-mail: apfelbaum.3@osu.edu

B. McMurray

Department of Psychology and Delta Center, University of Iowa, Iowa, IA, USA
Keywords Categorization · Speech perception . Expectation-relative processing $\cdot$ Predictive coding $\cdot$ Exemplar models · PDP models

\section{Introduction}

Research on perceptual categorization has often emphasized debates about the architecture of categorization, or how perceptual inputs are mapped to categories. Such debates have centered on issues like the representational format of categories (e.g., Minda \& Smith, 2002; Nosofsky, Kruschke, \& McKinley, 1992; Smith, 2002), and the possibility of distinct systems that perform these mappings (e.g., Love, Medin, \& Gureckis, 2004). This approach has often ignored the impact of the processes that encode the perceptual cues ${ }^{1}$ that serve as the basis of categorization. In this framing, the central question has been how people categorize or assign labels to these cue-values, whereas what people categorize (the content at the level of cues) has been rarely investigated (though see Riesenhuber \& Poggio, 2000).

If the goal is to characterize the general system and how it deals with information regardless of domain, the architecture is clearly an important emphasis. Such investigations benefit from using categories that are well-defined by arbitrary dimensions like color, brightness and orientation, or auditory cues like frequency in audition (e.g., Maddox, Bohil, \& Ing, 2004; Medin \& Schaffer, 1978; Nosofsky, 1988; Posner \& Keele, 1968; Zaki \& Nosofsky, 2007). Crucially, in these studies, category membership is determined straightforwardly by the orthogonally varied perceptual features or by cue

\footnotetext{
${ }^{1}$ Here, we define a cue as a continuous measurement of some aspect of the input (e.g., color, frequency), and the perceptual encoding refers to how these pre-categorical cue-values are identified and represented.
} 
values with a fairly unambiguous boundary (even if defined in a multidimensional space).

Such studies have proved successful at elucidating many components of categorization, and their findings often coincide with categorization in more natural categories like faces (Goldstone, Steyvers, \& Rogosky, 2003). However, extending these findings to more ecological domains in areas like speech perception is not straightforward. In speech perception, it is argued widely that the initial encoding of perceptual cues is not peripheral to the problem of categorization, as this cue encoding must be complex: cue-values cannot be identified straightforwardly, and the particular cue-values of a stimulus may derive from multiple factors simultaneously. Thus, there is debate about the nature of the perceptual cues and the process of encoding these cues. Consequently, the nature of the processes that work at the level of cue-values may interact with categorization architecture, and it may be necessary to understand such processes in order to fully capture the problem.

\section{Speech}

In speech perception, the informational structure of the cues is important: speech categories are defined by variable, complex and multiply determined perceptual properties. Phoneticians have measured many cues in the speech signal to identify those that relate to speech segment identity. This body of work suggests that, unlike the clean cases employed in more basic work on categorization, acoustic/phonetic cues are more ambiguous at multiple levels. Speech categories are highly overlapping in cue-space (Hillenbrand, Getty, Clark, \& Wheeler, 1995; Miller, Green, \& Reeves, 1986), the cues that comprise them are highly variable (Hillenbrand, Clark, \& Nearey, 2001), and speech categories often rely on the use of a large number of cues (Hillenbrand et al., 1995; Jongman, Wayland, \& Wong, 2000; Lisker, 1986; McMurray \& Jongman, 2011). Often, the relevant cues are asynchronous, requiring a listener to combine information across time-points in a word (Apfelbaum, Bullock-Rest, Rhone, Jongman, \& McMurray, 2014; Jongman et al., 2000; McMurray, Clayards, Tanenhaus, \& Aslin, 2008; Rhone \& Jongman, 2012; Summerfield, 1981; Toscano \& McMurray, 2012). Crucially, in many cases, individual cues vary as a function of many factors simultaneously (Cole, Linebaugh, Munson, \& McMurray, 2010; McMurray \& Jongman, 2011; Mermelstein, 1978; Whalen, 1989). For example, the length of a vowel can be affected simultaneously by the identity of the vowel and talker, whether the syllable is stressed, speaking rate, and voicing of neighboring consonants. As a result there are few invariant markers (in single cues or across multiple cues) that define phonetic categories (McMurray \& Jongman, 2011; Ohala, 1996).

This rampant variance and covariance in the speech signal - the well-known problem of lack of invariance - makes categorization in speech perception a difficult problem. As a result, focusing solely on the architecture of categorization (e.g. prototypes vs. exemplars) may not be fruitful when done in independently of the issue of how speech cues are processed and encoded, particularly given that multiple factors simultaneously influence most speech cues.

Speech perception also offers a unique opportunity to examine the relationship between cue processing and categorization. Phonetic analysis offers concrete ways to measure and quantify information in the signal, and this in turn offers the possibility of a clearer approach to differentiating categorization architectures (e.g. prototypes and exemplars) that are often difficult to distinguish, using a set of cues (the information) derived from the statistics of natural exemplars. Specifically, we can start with a set of cue measurements of actual speech (as opposed to contrived laboratory categories or unquantifiable natural visual categories). Using such measurements, we can simultaneously investigate continuous cueencoding and architectural models of how those cues are mapped to categories. In particular, by orthogonally varying cue-encoding and categorization strategies in computational models, we can identify the unique contributions of each in coping with variability in the speech signal.

This is particularly important given competing claims about the information available in the signal. For example, while most approaches agree on the lack of invariant mappings in the signal, as we discuss shortly, exemplar models argue that this can be overcome with architectural features of categorization, while relative cue encoding or predictive coding models argue for solutions at the cue-encoding level. The fact that these models solve the problem at different levels of the system makes it difficult to determine what is necessary to solve the problem of lack of invariance. Orthogonally varying aspects of cue encoding and the architecture of categorization in a computational model may help isolate the role of perceptual encoding, and this in turn can help constrain the realm of possible categorization systems underlying speech perception, in a way that potentially has implications for categorization in other domains.

We gauged how different forms of information affect different classes of categorization systems. In reviewing these systems, it important to note that theories of speech perception differ on many dimensions, but, for the present purposes, we focus on differences in their approach to cue encoding where there appear to be three broad approaches: categorical encoding, continuous acoustic encoding, and relative encoding.

\section{Categorical encoding}

Early accounts of speech suggested that cue encoding was warped by the presence of categories to minimize differences within a category and exaggerate differences across 
categories: so-called categorical perception (Liberman \& Cooper, 1967; Liberman, Harris, Hoffman, \& Griffith, 1957). This implied a strong isomorphism between cueencoding and categorization. However, a wealth of later studies undermined the evidence for categorical perception (Carney, Widin, \& Viemeister, 1977; Gerrits \& Schouten, 2004; Massaro \& Cohen, 1983; Pisoni \& Tash, 1974; Schouten, Gerrits, \& van Hessen, 2003), and ultimately supported a more continuous representation of the signal (Frye et al., 2007; Toscano, McMurray, Dennhardt, \& Luck, 2010), which is later mapped onto categories (Myers, Blumstein, Walsh, \& Eliassen, 2009). Thus, we do not consider this form of cue encoding further here.

\section{Continuous acoustic encoding}

Building on this continuity in cue encoding, a number of theories posit that listeners encode veridical representations of acoustic information. Historically, such approaches grew out of work identifying invariant acoustic cues for several phonological distinctions (Blumstein \& Stevens, 1979, 1980; Lahiri, Gewirth, \& Blumstein, 1984). These invariance approaches suggested that listeners ignore variable aspects of the signal (variability due to talker, coarticulation, speaking rate and the like) and focus only on cues that are invariant across productions. The information content for categorization in this account can thus be computed directly from the raw acoustic signal. While phonetic analyses searching for invariant cues showed early promise, particularly for place of articulation (Blumstein \& Stevens, 1979, 1980; Lahiri et al., 1984), the cues derived from such analyses often failed to discriminate phonemes at all syllable positions (Blumstein \& Stevens, 1979).

To cope with this, more sophisticated compound cues (cues constructed from multiple measurements), such as relative formant frequencies (Ladefoged \& Broadbent, 1957), duration ratios (Pind, 1995; Port \& Dalby, 1982) or locus equations (Sussman, Fruchter, Hilbert, \& Sirosh, 1998), often fare better by implicitly accounting for contextual factors through the dynamics of single cue values. For example, the second formant frequency (F2) at syllable onset is a cue for place of articulation, but is also affected by the following vowel. F2 might be more effectively used to identify place of articulation if it is first subtracted from the F2 at the vowel (Sussman et al., 1998). Critically, this operates purely on information in the auditory signal.

In a similar vein, Holt, Kluender, Lotto and colleagues have argued that basic auditory processes like contrast use context to interpret cues like formants and durations (Coady, Kluender, \& Rhode, 2003; Holt, 2006; Kiefte \& Kluender, 2008; Kluender, Coady, \& Kiefte, 2003; Lotto \& Kluender, 1998; though see Viswanathan, Fowler, \& Magnuson, 2009); for example, a low tone preceding a consonant causes listeners to hear the formant values of that consonant as higher, shifting its perceived place of articulation. Again, while this implicitly includes contextual information, it is done solely with regard to other cue-level information.

Compound cues like locus equations have proven effective for only a handful of phonological contrasts, and the more general auditory contrast accounts have not been applied explicitly as a solution to the problem of lack of invariance. Given the lack of invariant cues (single or compound) for numerous phoneme categories (Hillenbrand et al., 2001; McMurray \& Jongman, 2011), an alternative is that speech perception relies on more than a few cues, i.e., that massive amounts of information must be used simultaneously, as in exemplar models (Goldinger, 1998) and cue integration models like FLMP and NAPP (Nearey, 1990; Oden \& Massaro, 1978; Toscano \& McMurray, 2010). Here, acoustic information is encoded in a fairly raw form, but the use of many cues alleviates difficulties arising from only partially diagnostic individual cues. This is closer to how most models of categorization in other domains operate, using multiple dimensions to classify stimuli. The fundamental idea is that, while there may be no clear boundary for disambiguating categories along a single cue-dimension, such a boundary is possible in a high-dimensional space (Nearey, 1997). This ensures more context-invariant phoneme recognition despite the variability of cues from different talkers and contexts. Further, this fairly high dimensional representation of the input enables somewhat simple prototype architectures to map this cue-space onto categories (e.g., Oden \& Massaro, 1978; Nearey, 1990).

Multiple cue approaches have often been critiqued for ignoring the fact that cue values can be influenced by several factors simultaneously. For example, the frequency of the first formant (F1) is a useful cue for vowel identity, yet there is also substantial variability in it. Critically, much of this variability is not noise (as much as $90 \%$ in some studies: Cole et al., 2010); it is lawfully related to talker and consonant differences. Cue integration models argue that integrating these noisy estimates of F1 with secondary cues like F2, pitch (F0) and duration may be useful. However, factors like talker that influence F1 also add variability to F2, F0 and duration. Thus, simply integrating multiple cues may be insufficient for dealing with variability when it is caused by multiple factors influencing the same set of cues (Whalen, 1992).

Exemplar models attempt to solve this issue by embracing variability (Creel, Aslin, \& Tanenhaus, 2008; Goldinger, 1998; Hawkins, 2003). Exemplar-based speech encoding maintains that listeners have veridical encodings of raw acoustic input and perform a holistic match of the input to previously heard utterances. When a speech segment's acoustic realization varies as a function of context, exemplar models use previous tokens from similar contexts to identify the stimulus. For example, even as F1 may be lower for a 
particular talker, by matching the entire utterance to other similar utterances in memory (many of which may have been spoken by that talker or similar-sounding talkers with similarly low $F 1$ values) the lawful relationships between cues can be accounted for.

Support for exemplar-based phoneme recognition comes from studies showing that listeners are faster and more accurate in recognizing words spoken in familiar voices (Bradlow, Nygaard, \& Pisoni, 1999; Creel et al., 2008; Goldinger, 1998; McLennan \& Luce, 2005). However, many researchers have been hesitant to adopt this approach due to concerns over memory demands, and exemplar models have not yet been applied directly to the problem of categorizing speech from real phonetic measurements, leaving it an open question whether they can harness the variability in the speech signal to perform human-like phoneme recognition.

To briefly summarize, the earliest approaches (invariance, compound cue, auditory accounts) stressed the information available in the signal and assumed some simple mapping to categories (e.g., a decision boundary). Later approaches, like the exemplar and prototype accounts, agreed on a very high dimensional representation of the signal, but differ on the architecture of categorization. However, all of these approaches utilize a more or less continuous cue encoding that is largely a bottom-up product of the signal.

\section{Predictive coding or expectation-relative coding}

A number of more recent models suggest that listeners perceive speech stimuli relative to expectations driven by other cognitive decisions and knowledge of context effects ${ }^{2}$ (Cole et al., 2010; Johnson, Strand, \& D'Imperio, 1999; McMurray, Cole, \& Munson, 2011; McMurray \& Jongman, 2011; Smits, 2001a, b; Whalen, 1989). In these approaches, the system makes a determination about some aspect of context (e.g., the identity of the talker or the neighboring phoneme) and uses this information to guide how other cues are encoded or used. For example, listeners may encode formants as lower than expected for a woman, or higher than expected after a labial. Thus, perception is a product of both what is in the signal and expectations that are partially the result of longterm learning.

This use of context could occur at the level of perception or at a later categorization stage. Indeed, several theories suggest that similar context effects pervade multiple levels of

\footnotetext{
$\overline{2}$ Although compound cues and locus equations are a form of relativization, we describe them as examples of absolute or raw-cue encoding. This is because the compounds are constructed directly from two cue values (e.g., the difference or ratio). In contrast, the relative cue approaches assume that cues are computed relative to some kind of cognitive process (e.g., talker identification, identifying a neighboring phoneme), not just on the basis of bottom-up cue combination from veridically encoded acoustic information.
}

cognitive processing (Carandini \& Heeger, 2011; Clark, 2012). We emphasize instances wherein the effects operate at an early, perceptual level in our descriptions; we made this choice as it accords with recent work on relative encoding (Cole et al., 2010; McMurray et al., 2011; McMurray \& Jongman, 2011), because it fits well with evidence of continuous information integration, and because it allows an easy orthogonalization of the question of relative vs. absolute cues and the architecture of categorization. However, a similar relativization can also be achieved by conditioning categorization decisions on context and leaving cue estimates unchanged (Smits, 2001b). The precise mechanisms of relativizing cues against such expectations are an open empirical question, so the exact locus of such effects remains a matter of speculation. Further discussion of the different possible loci of expectation-relative (ER) encoding and our preference for cue-level recoding is provided in the General discussion.

Expectation-relative encoding in speech perception is akin to predictive coding (see McMurray, Rhone \& Hannaway, 2015; Clark, 2013 for a review), which has become an important idea in vision and motor control. In predictive coding, perception proceeds through constant prediction of input and evaluation of these predictions against the actual form of the input. Predictions are formed on the basis of context, creating a means for context to affect the interpretation of the bottomup perceptual signal. Critically, as in ER encoding schemes, the informational basis of perception is the difference between expected and actual events. For example, event perception appears to rely on predictions about upcoming information; violations of these expectations signal event boundaries (Zacks, Speer, Swallow, Braver, \& Reynolds, 2007). Similar forms of predictive coding have been suggested for speech perception (Gagnepain, Henson, \& Davis, 2012) as well as for higher-level aspects of language (Pickering \& Garrod, 2004). There is also neuroimaging evidence that context can impact activation in lower-level sensory areas (typically reducing activity for expected events), providing some support for this idea (von Kriegstein, Smith, Patterson, Kiebel, \& Griffiths, 2010; Zacks et al., 2007). The concepts of ER encoding and predictive coding are quite similar, if not isomorphic. We continue to use the term ER encoding here for consistency with prior work on speech perception, and return to the deep overlap between these ideas in the General discussion.

Within speech perception, the first instantiation of these ideas derives from adaptive resonance models of speech perception, ARTWord (Grossberg \& Myers, 2000) and ARTPhone (Grossberg, 2003). In these models, phonemes are recognized in running speech by developing resonances based on both bottom-up acoustic information within the phoneme, as well as top-down information from judgments of other phonemes. This interaction between sources of information allows the listener to incorporate judgments of surrounding context into her perception. This system nicely 
accommodates things like coarticulation, phoneme restoration and backward context effects. In the ART models, rather than mapping between acoustics and phoneme categories veridically, listener's judgments are colored by surrounding context, even if that context occurs after the phoneme in question. These ART models provide compelling explanations for several instances of context-specificity in speech perception (although these models perform such context-relative encodings only on the basis of neighboring acoustic information, not on the basis of higher level information, such as talker gender or other social expectations).

Moreover, there is also mounting empirical evidence that expectations affect phoneme judgments in a number of ways. When listeners hear an ambiguous sound between $/ \mathrm{s} /$ and $/ \mathrm{g} /$, but are told it was spoken by a female (e.g., a video of a female producing it), they are more likely to classify the token as $/ \mathrm{J} /$, but if they are told it was spoken by a male, they hear an $/ \mathrm{s} /$ (Johnson et al., 1999; Strand, 1999). Similar effects have been found using expectations from talker age (Drager, 2011), dialect (Niedzielski, 1999), and expectations of hearing a single or multiple talkers (Magnuson \& Nusbaum, 2007). Changing response labels to encourage expectations of different manners of articulation also affects how listeners treat stimuli (Carden, Levitt, Jusczyk, \& Walley, 1981), and even presenting stuffed animals that are associated with certain dialects (e.g. kangaroos for Australian dialects) leads listeners to identify speech tokens relative to how they expect talkers of that dialect to speak (Hay \& Drager, 2010). Expectations generated within the speech signal also affect identification; when a vowel from another talker or vowel context is spliced onto a fricative, listeners are less adept at correctly identifying the fricative (Apfelbaum et al., 2014), even when the bottomup cues all match the fricative in question. Listeners' phonetic judgments are thus highly contingent on how they expect a talker to produce speech segments.

However, evidence that listeners use expectations when such cognitive factors are not as salient has been mixed. A series of studies dating back to Mermelstein (1978; Nearey, 1990, 1997; Smits, 2001a, b; Whalen, 1989, 1992) have looked at the contingency of different simultaneous decisions about the same speech cues. For example, in the words bad/ bed/bat/bet, F1 is a cue to both vowel identity and word-final voicing. Absolute cue encoding predicts that this overlap is simply used independently for both phonetic judgments (Mermelstein, 1978; Nearey, 1990) - a lowered F1 is simultaneously a cue that the vowel is an $/ \varepsilon /$, and that the consonant is voiced. In contrast, in contingent accounts (Smits, 2001a, b; Whalen, 1992) listeners first identify the vowel as an $/ \varepsilon /$ (for example); then, that low F1 is considered relative to typical values for $/ \varepsilon$ / (i.e. its lowered frequency is attributed to the vowel), so its low value has less of an effect on voicing judgments. A number of studies have adopted this logic to fit computational models of categorization (usually variants of a prototype model) to listeners' categorization functions, but have yielded little consensus. Indeed, identical data have been analyzed and presented as support of integrating absolute cues (Nearey, 1997), and as evidence of ER encoding (Smits, 2001a, b; Whalen, 1992).

More recently, McMurray and Jongman (2011) conducted a thorough comparison of raw and ER encoding using a large corpus of measurements of natural fricatives (based on Jongman et al., 2000). Twenty-four cues to fricative identity were measured for 2,880 tokens of the eight fricatives of English. These cues included all of the known cues to fricative perception, as well as several new ones. This corpus spanned six different vowels and 20 talkers, and thus included substantial overlapping variability. A subset of these tokens was classified by listeners to provide a baseline for model evaluation. These cue values were then used as input to models of fricative categorization, and model performance was compared to human identification (both in terms of absolute performance level and in the pattern of errors). Crucially, the same categorization model (a prototype model similar to Nearey, 1990, 1997; Oden \& Massaro, 1978), was used with both raw and ER cues as input. The raw cue model used the measurements for all 24 cues for each fricative as input, simulating the most comprehensive multiple-cue invariance model possible. To simulate ER encoding, a hierarchical linear regression was used to remove effects of talker and vowel from the acoustic cue measurements prior to submitting the data to the categorization model. The ER model was then compared to a model using raw acoustic information. The model using ER cues yielded much better performance than the raw cue model; whereas listeners averaged $91.2 \%$ correct, the absolute cue model performed much worse $(79.2 \%$ $\left.85.0 \%{ }^{3}\right)$, but the ER cue model performed in the right range $(87.0 \%-92.9 \%)$. The ER model also better matched listeners' pattern of errors; for example, this model showed the best identification performance for fricatives preceding $/ \mathrm{u} /$ (as did listeners), whereas the raw cue model showed its worst performance for these fricatives. Thus, holding the categorization mechanism constant, this model suggests that ER cues can offer a close quantitative and qualitative fit to human data, whereas the raw cue model fell short.

At one level, these benefits of ER encoding are unsurprising; the use of expectations in this way can be conceptualized as a form of noise reduction on the signal, which ought to make the categorization problem more straightforward. Removing variance is a simple way to improve accuracy for the model. However, this oversimplifies things a bit, as the noise reduction is not geared to the problem of fricative identification, but rather to the variance created by talker and vowel-it was entirely possible that reducing the noise due to

\footnotetext{
${ }^{3}$ As we will discuss, there are two ways to compute performance from such models, so we report performance with a range of values.
} 
these other factors would eliminate information relevant to fricative identification or have no bearing on it. Moreover, there is wide debate about whether listeners engage in this form of noise reduction; many researchers continue to assert that raw information is used by listeners, and that some cue or combination of cues is present in the raw signal that is sufficient to make all phonetic distinctions (e.g., Juneja \& EspyWilson, 2008; Stevens \& Keyser, 2010; Stevens, 2002). Moreover, whereas it may not be surprising that ER encoding can improve classification accuracy, it is not necessarily a given that that will yield improved fit to patterns of human performance, especially patterns of human errors. If relativizing cues for effects of context in the model leads to close fits to human performance, this suggests that listeners may engage in similar forms of ER encoding. Thus, while this scheme may appear fairly intuitive, it is by no means obvious, but rather one of a wide number of theoretical approaches that are still under debate.

\section{The present study}

The evidence from previous models of ER encoding (McMurray \& Jongman, 2011) shows better fit to human data than models based on raw cues when assuming a prototypestyle categorization architecture. However, there remains debate over whether such an architecture is appropriate for representing human categorization with more abstract (typically visual) categories (e.g., Medin \& Schaffer, 1978; Smith \& Minda, 1998; Zaki \& Nosofsky, 2007); other classes of models may be able to handle the variability of speech within the categorization architecture. For example, as we described, exemplar models may implicitly account for it by matching cues more holistically to exemplars, using surface variability to aid this matching. Thus, although ER encoding or predictive coding is a promising candidate explanation for how listeners cope with and utilize acoustic variability, establishing this requires a more thorough investigation of how relative and absolute encoding of information interact with various categorization architectures. Specifically, it remains to be investigated whether more advanced categorization architectures, like exemplar models, can accommodate the highly variable speech signal using raw acoustic cues. That is, it is not currently known whether the apparent necessity of relative cues for accounting for listener performance is a consequence of McMurray and Jongman's (2011) fairly basic categorization architecture; other architectures may produce human-like performance using raw acoustic information, as posited by a large proportion of speech researchers over the last 50 years.

Two strong candidates for speech categorization architectures that may mimic human performance using raw acoustic information are exemplar models and multi-layer parallel distributed processing (PDP) models trained with back-propagation. Neither has been applied extensively to speech.
In exemplar models, surface variability (i.e., acoustic variability that is not necessarily related to phonetic categories) can differentiate similar tokens by helping the system identify a token based on experience with tokens in similar contexts. Recoding information to eliminate context effects could thus prove disastrous for exemplar models. However, exemplar models have not been implemented extensively for speech (though see Goldinger, 1998), and none have been used with real phonetic measurements. Such models have several free parameters, like the temperature of the similarity function; therefore their predictions in this regard are not straightforward. Thus, part of investigating this question requires us to examine the range of implementational options, which have not been examined in the context of speech categorization.

PDP models - for our purposes, multi-level feedforward networks using non-linear hidden units, trained with backpropagation —offer a very different approach. These have also not been applied extensively to speech perception (though see Elman \& Zipser, 1988). However, as we describe, they are formally related to a number of prototype models of speech like FLMP (Oden \& Massaro, 1978) and NAPP (Nearey, 1990). In PDP models, units are connected with weights that are adjusted to best solve the classification problem at hand. In a speech perception PDP model, units representing acoustic inputs are connected via hidden layers to units representing speech categories. By learning the ideal set of weights to connect these layers, the model learns how to translate acoustic information into categorical decisions.

The PDP architecture, and specifically the fact that hidden units are allowed to discover the necessary substructure of a problem, allows these models to behave flexibly, imitating behaviors of other categorization architectures. Commonly used activation functions for hidden and output units allow such networks to approximate logistic regression, capturing similar processes to existing prototype models of speech (McMurray \& Jongman, 2011; Nearey, 1997; Oden \& Massaro, 1978; Smits, 2001b). However, the hidden units allow such networks to discover higher-order regularities when simple invariant cues are unavailable, and to separate categories that are not linearly separable (Rumelhart, Hinton, \& Williams, 1986), allowing them to act as "universal function approximators" (Hornik, Stinchcombe, \& White, 1989). Thus, these models significantly exceed the capacity of approaches like logistic regression.

Hidden units can simultaneously represent useful generalizations (akin to phonemes or features, though rarely at this exact level), but also show sensitivity to specific exemplars or regions of exemplar space, especially when many hidden units are available. Thus, such models can act as a hybrid of prototype and exemplar models: in portions of the taskspace where exemplars are useful, the weights can encode surface-form information. However, in situations where greater consistency exists, PDP models can store more abstract 
information. Thus, these models, like exemplar models, may not need ER encoding. If the raw acoustic signal is sufficient to allow effective categorization, this powerful architecture offers the promise to find the appropriate mappings. Noise reduction through ER encoding would thus obfuscate the signal used by the models, potentially leading the models to performance that differs from humans (even if quite accurate).

\section{Goals}

While computational studies have examined the information content available to support categorization (Cole et al., 2010; McMurray \& Jongman, 2011), or have compared a limited number of categorization architectures given a common input (Nearey, 1989, 1997; Smits, 2001a, b), our goal was to cross information content with categorization architecture.

We started with the large corpus of phonetic measurements of the eight English fricatives (/f, v, $\theta, \delta, s, z, \int, z /$ ) first developed by Jongman et al. (2000) and extended by McMurray and Jongman (2011). This included 24 measurements of 2,880 exemplars produced by 20 talkers in a range of vowel contexts (Jongman et al., 2000). We adopted McMurray and Jongman's (2011; see also Cole et al., 2010) $\mathrm{C}$-CuRE approach to converting these cues to ER cues. We did not simulate the process of generating the expectations used in ER processing, but instead used regression to accomplish this before submitting the inputs to the categorization model. This enabled us to train a series of exemplar and PDP models using either raw or ER cues from this corpus and to assess them against listener performance, without altering the architecture between models; by performing the ER encoding outside of the categorization model, we hold the architecture constant and vary the form of the input. Specifically, we asked whether ER information is consistently beneficial across architectures, or if some architectures (or variants) perform better when using raw information. If even those categorization architectures that rely on context-rich information are improved by ER information encoding, this would support theories that argue that information is encoded relative to expectations and context during speech perception. This would also demonstrate the value of considering information content in addition to architecture when investigating categorization processes.

These questions have already been posed using traditional prototype models (McMurray \& Jongman, 2011), which showed support for ER encoding. Here we investigate exemplar and PDP models, which may be better suited to using the raw acoustic signal. There are no clear models of speech perception in either framework on which to build. The closest analogues are
Goldinger's (1998) adaption of MINERVA and Elman and Zipser's (1988) PDP model. However, Goldinger did not use real speech cues and did not explore the range of implementational options we were interested in here, and Elman and Zipser used actual acoustic signals as input, which, though admirable, makes it difficult to compute ER cues. Thus, we developed new models of speech perception for each architecture. Although our primary goal was to gauge the necessity of ER processing, developing these models had the added benefit of determining how well these architectures can account for speech perception using real speech data, and yielded some insight as to how these types of categorization architectures play out in speech. A crucial part of this investigation is a systematic exploration of the parameter-spaces of each architecture (Apfelbaum \& McMurray, 2011; Pitt, Kim, Navarro, \& Myung, 2006). While these models were too different to compare to each other (as in Pitt et al., 2006), a parameterspace search is crucial for ensuring that our findings generalize across different parameter sets, and that we are comparing the "best" models under different training conditions.

In the remainder of this paper we first describe the structure of the training data that was common to both models. Next we describe the implementations of each model, and their results.

\section{The corpus}

The corpus of cue-values was the same as those reported in McMurray and Jongman (2011, and publicly available in the online supplement for that paper). Fricative discrimination is thought to rely on a wide range of different cues, including information during both frication and in the vowel, and including duration, amplitude and spectral components (Jongman et al., 2000).

The fricatives were produced by 20 talkers ( 10 male). Each talker produced every English fricative (/f, v, $\Theta, \partial, \mathrm{s}, \mathrm{z}, \int, \mathrm{z} /$ ) in syllable-initial position followed by each of six vowels (/i, e, $æ, \mathrm{a}, \mathrm{o}, \mathrm{u} /$ ), and repeated three times, for a total of 2,880 tokens. Of these, measurements of all 24 cues were available for 2,873 tokens; the remaining seven had missing cue-values set to cell-means (talker $\times$ fricative $\times$ vowel). The measured cues include all cues in both the frication and in the vowel thought to be useful for distinguishing fricatives. Cues that require compounding across multiple cues or using relative values were not included [though McMurray \& Jongman (2011) found similar results when using such cues). A full list of cues is shown in Table 1. All cue values were normalized as $Z$-scores (relative to the overall mean and SD of the entire dataset). 
Table 1 Description of the cues measured for the fricatives included in the models

\begin{tabular}{|c|c|c|}
\hline Cue & Description & Location \\
\hline Peak Frequency & $\begin{array}{l}\text { Frequency with } \\
\text { highest amplitude }\end{array}$ & Frication \\
\hline Frication Duration & Duration of frication & Frication \\
\hline Vowel Duration & $\begin{array}{l}\text { Duration of } \\
\text { vocalic portion }\end{array}$ & Vowel \\
\hline Frication RMS & Amplitude of frication & Frication \\
\hline Vowel RMS & $\begin{array}{l}\text { Amplitude of } \\
\text { vocalic portion }\end{array}$ & Vowel \\
\hline F3 amplitude (frication) & $\begin{array}{l}\text { Amplitude of frication } \\
\text { in F3 range }\end{array}$ & End of frication \\
\hline F3 amplitude (vowel) & $\begin{array}{l}\text { Amplitude of F3 } \\
\text { in vowel }\end{array}$ & Onset of vowel \\
\hline F5 amplitude (frication) & $\begin{array}{l}\text { Amplitude of frication } \\
\text { in F5 range }\end{array}$ & End of frication \\
\hline F5 amplitude (vowel) & $\begin{array}{l}\text { Amplitude of F5 } \\
\text { in vowel }\end{array}$ & Onset of vowel \\
\hline Low Frequency Energy & $\begin{array}{l}\text { Mean RMS } \\
\text { below } 500 \mathrm{~Hz}\end{array}$ & Frication \\
\hline Pitch & Fundamental frequency & Vowel onset \\
\hline First Formant & First formant frequency & Vowel midpoint \\
\hline Second Formant & Second formant frequency & Vowel midpoint \\
\hline Third Formant & Third formant frequency & Vowel midpoint \\
\hline Fourth Formant & Fourth formant frequency & Vowel midpoint \\
\hline Fifth Formant & Fifth formant frequency & Vowel midpoint \\
\hline Spectral Mean (2) & Spectral mean, 2 windows & $\begin{array}{l}\text { Frication onset, } \\
\text { midpoint }\end{array}$ \\
\hline Spectral Variance (2) & $\begin{array}{l}\text { Spectral variance, } \\
2 \text { windows }\end{array}$ & $\begin{array}{l}\text { Frication onset, } \\
\text { midpoint }\end{array}$ \\
\hline Spectral Skewness (2) & $\begin{array}{l}\text { Spectral skewness, } \\
2 \text { windows }\end{array}$ & $\begin{array}{l}\text { Frication onset, } \\
\text { midpoint }\end{array}$ \\
\hline Spectral Kurtosis (2) & $\begin{array}{l}\text { Spectral kurtosis, } \\
2 \text { windows }\end{array}$ & $\begin{array}{l}\text { Frication onset, } \\
\text { midpoint }\end{array}$ \\
\hline Transition Mean & $\begin{array}{r}\text { Spectral mean } \\
\text { at transition }\end{array}$ & $\begin{array}{l}\text { Frication offset } \\
\text { and vowel onset }\end{array}$ \\
\hline Transition Variance & $\begin{array}{l}\text { Spectral variance } \\
\text { at transition }\end{array}$ & $\begin{array}{l}\text { Frication offset } \\
\text { and vowel onset }\end{array}$ \\
\hline Transition Skewness & $\begin{array}{l}\text { Spectral skewness } \\
\text { at transition }\end{array}$ & $\begin{array}{l}\text { Frication offset } \\
\text { and vowel onset }\end{array}$ \\
\hline Transition Kurtosis & $\begin{array}{l}\text { Spectral kurtosis } \\
\text { at transition }\end{array}$ & $\begin{array}{l}\text { Frication offset } \\
\text { and vowel onset }\end{array}$ \\
\hline
\end{tabular}

To simulate ER cue encoding, cues were conditionalized with respect to vowel and talker ${ }^{4}$ context using linear regression (McMurray \& Jongman, 2011; Cole et al., 2010;

\footnotetext{
${ }^{4}$ This presumes that listeners can identify the vowel and talker of the tokens. Although listeners may not be familiar with specific talkers, McMurray \& Jongman (2011) found very similar patterns when using talker gender rather than talker identity. A more comprehensive model might include bidirectional influences between fricative and vowel identification, with partial activations mutually informing each other. However, for the purposes of straightforward explication, we make the simplifying assumption that listeners identify the vowel and talker accurately without using fricative identity.
}

McMurray et al., 2011). First, individual regression equations were developed that predicted each cue-value from talker (19 dummy codes) and vowel (5 dummy codes). We then took the residuals from these regression equations and standardized them to put all dimensions on the same scale. This resulted in cue values that signal how each cue differs from what would be expected given the talker and vowel context. Critically, these regressions did not include the fricative target as a factor, and the equations were not estimated with this as a factor-thus, they were not optimized to the problem of interest.

Of the 2,880 tokens in the corpus, 240 tokens ( 8 fricatives $\times$ 10 talkers $\times 3$ vowels) were also used in a perception experiment (McMurray and Jongman, 2011), in which 20 participants identified the onset fricative of each token. Thus, models were trained on 2,640 tokens (330 from each fricative category) and tested on the 240 tokens for which perceptual data were available.

\section{Exemplar models}

Implementation

\section{Architecture}

Exemplar models make use of a memory architecture in which individual episodes are remembered in high fidelity (Goldinger, 1998; Pierrehumbert, 2003; see also, Kruschke, 1992; Medin \& Schaffer, 1978; Nosofsky, 1986; Nosofsky et al., 1992 for representative models in visual categorization). To categorize an incoming stimulus, this item is compared against stored episodic traces to determine how similar it is to past exemplars. Exemplars are associated with their category, so the set of similar exemplars can be used to categorize the input token. In exemplar accounts applied to speech, phonemic and talker-specific information do not play differential roles in the episodic traces (Goldinger, 1998). Rather than abstracting to a phonetic encoding of a word, listeners maintain acoustic detail in their long-term storage of speech sounds and words, and use these detailed memory traces to recognize new tokens. This categorization system presupposes raw acoustic encoding to allow matching of the input to previous exemplars. Our approach represented stored exemplars as vectors of cue-values across the 24 acoustic cues in the corpus. The collection of all such vectors (corresponding to each of the tokens) is the model's exemplar memory. A matching model using ER cues was constructed by using the 24 ER cue values (eliminating the effect of talker and vowel context).

To categorize a given token, we computed its similarity to the stored tokens using a metric akin to previous exemplar models (Nosofsky, 1986, 1988). First, we compared the incoming token to the cloud of stored exemplars by computing the distance between the vector for the current token and the 
vectors for the stored exemplars. However, not all cues should receive equivalent weight for this comparison, as listeners likely discover which cues are most useful through lifelong experience with speech. Thus, we assumed each cue had an independent weight (Nosofsky, 1986), to estimate the weighted distance (Eq. 1).

$d_{t c}=\sqrt[\tau]{\sum_{m} w_{m}\left|v_{m t}-v_{m c}\right|^{\tau}}$

Here, $t$ represents the test exemplar (the stimulus) and $c$ is the comparison exemplar in memory. Weights are represented by $w$, and cue measurement values are represented by $v$, for all cues ( $m$, from 1 to 24). The value of $\tau$ was a free parameter, as in Nosofsky (1986). A $\tau$ of 2 is typically predicted to be most effective for models incorporating integral dimensions, whereas a $\tau$ of 1 is more useful for separable dimensions. As in Nosofsky (1986), we compared tested models using the two values of $\tau$ to determine which provided a better account for the data. We next converted this distance value to a similarity value:

$S_{t c}=e^{-d^{r}{ }^{r}}$

These distances are scaled by the $r$ parameter, as in Nosofsky (1986). An $r$ of 1 is an exponential decay function, whereas an $r$ of 2 is a Gaussian. As with $\tau$, we treat $r$ as a free parameter and test which value better accounts for the data.

From this similarity measure, we next computed the summed similarity of the token to each of the comparison categories (or rather, each cloud of exemplars that comprise a category):

$S_{t h}=\sum_{x} S_{t x}$

Here $h$ represents the fricative category for which the similarity is being computed and $x$ is the index from 1 to $n$ representing each individual exemplar. This was done for each of the eight categories.

This vector of eight continuous similarities represents how much the current token resembles the various tokens in each of the clouds of exemplars for the eight fricative categories. To convert this to a probabilistic choice, the similarity score for each category was divided by the sum of all similarity scores, yielding probabilities for the eight categories that sum to 1 :

$p(h \mid t)=\frac{S_{t h}}{\sum_{c} S_{t c}}$

Here, $C$ represents the set of comparison categories (the eight fricative categories).

In order to determine the correct weights for each model, a constrained gradient descent technique was used to maximize identification performance on the 2,640 tokens for which no perceptual data was available. These same weights were then used to test the models on the held-out tokens for which perceptual data was available. Weights were estimated separately for raw and ER models at each value of $\tau$. Initially, the weights for each cue were set to 1 . Using these weights, we estimated the probabilistic choice for each of the 2,640 training stimuli using the equations described above. For the purposes of training, if the model had the exact same token in memory, it could always reach the correct answer. Thus, when computing these probabilities, the current token was removed from the comparison cloud. The calculated probabilities for each test exemplar were then compared to the ideal responses - a vector consisting of all zeros for the incorrect categories, and a 1 for the category produced by the talker. We then used a gradient descent algorithm (the fmincon procedure of Matlab, using the interior point optimization technique) to minimize the difference (in terms of RMS error) between predicted model probabilities and perfect classification accuracy by adjusting cue weights; weights were constrained during learning to sum to 24 and to be greater than or equal to 0 . The final weights were saved and used in models of performance on the 240 testing stimuli not used during this fitting procedure.

\section{Predicting human performance}

Our final step in model evaluation was to compute the likelihood of each category for the 240 testing stimuli, and to convert the probabilities to something akin to human performance. This is applicable to both the exemplar model and the PDP model. As in McMurray and Jongman (2011), we used two methods to translate the probabilities into categorical responses. The first, the probabilistic rule, assumed that the calculated probabilities from these measures represent the true output probabilities for the model; that is, a stimulus with a $75 \%$ probability for $/ \mathrm{f} /$, a $20 \%$ probability for $/ \mathrm{e} /$ and a $5 \%$ probability for $/ \mathrm{s} /$ would be categorized as each phoneme with those same probabilities. Alternatively, the discrete-choice rule assumed that the category with the highest calculated probability will be selected every time; that is, in the above example, a category choice of /f/ would be given every time for that stimulus. As McMurray and Jongman (2011) discuss, while the "optimal" model is the discrete choice model (see also Nearey \& Hogan, 1986), human performance likely falls somewhere between these extremes. Rather than making assumptions about which form is more likely, we compare the range of response possibilities against the observed perceptual data (although log-likelihood is measured using the probabilistic rule).

These rules allowed us to compute for each of the 240 held out tokens a response range from the model. To capture model fit overall, we used a similar technique to McMurray and Jongman (2011). Each model output a vector of probabilities 
for each token signifying how likely it was to classify that token as a member of each fricative category (the probabilistic rule). Using these probabilities ${ }^{5}$ and the pattern of perceptual data, we can compute the likelihood of each individual token.

$L=\frac{N !}{A_{f} ! A_{s} ! \ldots A_{z} !} p_{f}^{A_{f}} p_{s}^{A_{s}} \ldots p_{z}^{A_{z}}$

Here, $N$ is the total number of responses in the perceptual data, $A$ is the number of times a category was given as an answer for that token, and $P$ is the probability of a given category to be output by the model. We can then take the $\log$ of each $L$ and sum these to give the log likelihood $(L L)$ of a given model. Models with larger $L L$ are a better fit to the perceptual data.

For several parameter settings (and for several parameter settings in the PDP models below), real-number $L L$ values could not be computed. For these models, on at least one token, the model was extremely confident in some categorization responses (making a probability for other responses extremely close to 0 ). If for this same token any participant ever chose one of these responses with a probability near 0 , the $L L$ for that trial could not be computed; that is, if a single participant pressed the wrong button or guessed, $L L$ brokedown as a measure of model goodness. Thus, we added a guessing rate parameter to the outputs (McMurray \& Jongman, 2011); this parameter adds a degree of noise to model outputs to ensure that categories rarely have probabilities too close to 0 to allow computation of $L L$. The probabilities were thus recalculated to incorporate this guessing rate:

$p_{\text {category }}=p_{\text {output }}\left(1-p_{\text {guess }}\right)+p_{\text {guess }}\left(\frac{1}{8}\right)$

This was computed using a range of different guessing rates $\left(P_{\text {guess }}\right)$, as we do not know a priori how often participants relied on guessing in generating their responses. We used rates ranging from very infrequent $\left(10^{-7}\right)$ to quite frequent $(0.1)$. This was used only for the $L L$ analyses. When response ranges are plotted and described we assumed a $P_{\text {guess }}$ of 0 , and accuracy data are presented without using a guessing rate adjustment.

\section{Simulations}

Each model was estimated from the training data and tested on the held-out stimuli for comparison with listeners' categorization (McMurray \& Jongman, 2011). For each simulation, this

\footnotetext{
${ }^{5}$ As the probabilistic output represents the lower-bound of model performance, using these values to compute $L L$ likely under-predicts model fit. However, more precise model estimates are not possible, and both raw and ER models face this same limitation.
}

was examined for both raw and ER cues (and weights were determined separately for each). $L L$ results are examined as a function of the free parameters, considering the models with the highest $L L$ across $P_{\text {guess }}$ values (although plotting of results is done using the pre-guessing-rate-adjustment data).

As with any large-scale model, there were a number of implementational assumptions that may have important theoretical consequences. For several of these choices, we examined multiple options. First, we considered how the value of $\tau$ on the difference measures affects performance (Nosofsky, 1986). We considered models with $\tau$ of 1 and 2; a $\tau$ of 2 is thought to be more appropriate for models with integral dimensions, where a $\tau$ of 1 is more appropriate for separable dimensions. We tested both as we were unsure a priori how integrally these dimensions are perceived. Second, we considered models with different values of the $r$ parameter on the similarity metric; we used values of 1 and 2 to examine exponential decay and Gaussian functions, respectively. Third, we considered how many exemplars should be considered in the comparisons; although the similarity metric scales comparisons such that far-away tokens have relatively little influence on the overall similarity, we investigated whether restricting the number of tokens in each comparison cloud affected performance. Finally, we considered whether the model should have access to stored exemplars from the same talker; this explores how the models generalize to novel talkers. ${ }^{6}$

Exemplar model 1: a representative model

As a first representative simulation, we examined a close analog of standard exemplar models. This model used a $\tau$ of 2 and an $r$ of 2, matching the most successful models presented in (Nosofsky, 1986). For this model, we compared the test tokens to every token in the training set, including other tokens from the same talker. We discuss the results of models with $P_{\text {guess }}$ values that provided the closest fit to human data (for the raw models, this was $P_{\text {guess }}=0.1$; for ER models it was $P_{\text {guess }}=0.01$ ), and plot results without the guessing rate adjustment.

Both the raw and ER models offered a close qualitative fit to the human perceptual data (Fig. 1). Both models predicted more accurate performance on sibilants (/s, z, $\left.\int, 3 /\right)$ than nonsibilants (/f, $\mathrm{v}, \Theta, \mathrm{\partial} /)$, and both models predicted poorest performance for $/ ð /$. However, the raw model predicted poorer

\footnotetext{
${ }^{6}$ While this model uses an equal-sized subset of comparison tokens from each of eight fricatives, one could also implement an exemplar model by considering the tokens within a certain distance in Euclidean space from the target, regardless of those tokens' categories (e.g., $60 \%$ of tokens within this range were $/ \mathrm{f} / \mathrm{s}, 20 \%$ were $/ \mathrm{e} /$ and so forth). Preliminary investigations showed that such models yielded results that were quite comparable to more traditional exemplar models, but also quite noisy, so these models are not included in this report.
} 

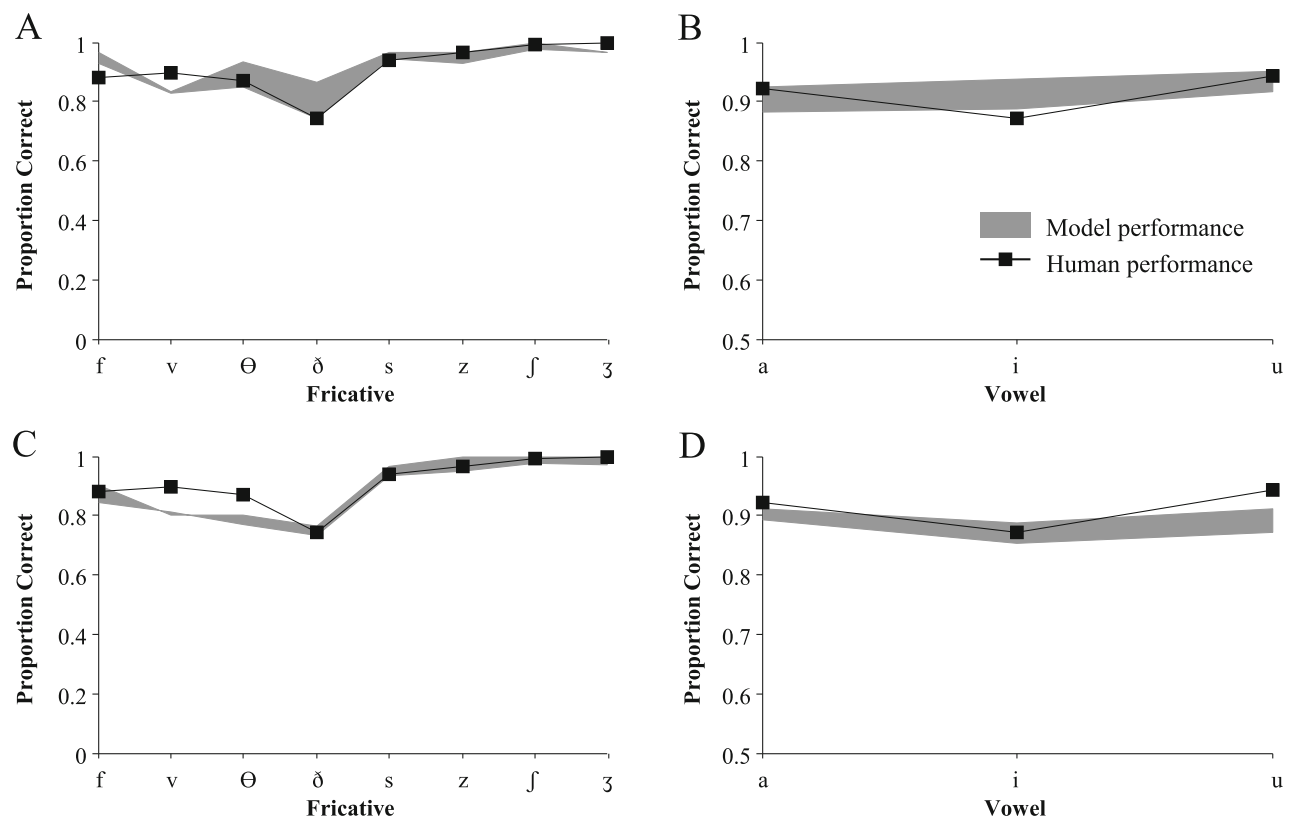

Fig. 1 a Output of the raw cues model compared to human performance by fricative. b Output of the raw cues model compared to human performance by vowel. c Output of the expectation-relative (ER) cues

performance than attested for $/ \mathrm{v} /$ and better performance than seen for /f/, Meanwhile, the ER model underpredicted performance for both $/ \mathrm{v} /$ and $/ \Theta /$. The models differed substantially in identification of the fricatives in different vowel contexts. Humans were least accurate in the context of an $/ \mathrm{i} /$; the ER model adhered to this pattern, but underpredicted performance for the $/ \mathrm{u} /$ context, whereas the raw model predicted poorest performance in the context of $/ \mathrm{a} /$.

Quantitatively, the raw model was more accurate overall than the ER model $\left(a c c_{\text {raw }}=93.2-93.8 \%\right.$; $a c c_{\mathrm{ER}}=87.2-$ $90.4 \%$ ). However, this accuracy benefit was not accompanied by an improved fit to human performance; instead, the ER model had a very slightly closer fit to the perceptual data $\left(L L_{\text {raw }}=-2,839 ; L L_{E R}=-2,830\right)$, suggesting that on a token-by-token basis, ER encoding more closely approximates human recognition. This is exemplified by the poor performance of the raw cue models to account for the effect of vowel context (Fig. 1b,d). More broadly, this confirms that, as discussed earlier, improved accuracy does not necessarily equate with improved fit to human performance; this finding is critical, as it shows that model decisions that are expected to improve accuracy (such as including a relativization step) do not necessarily mean that those models will better represent what humans do.

For this model, ER encoding offered a very slightly closer fit to human data than did raw encoding. Although this benefit is modest, it clearly rules out an advantage for raw cue encoding in this particular model. However, other architectural choices may reveal circumstances in which raw cue encoding excels; we thus

model compared to human performance by fricative. $\mathbf{d}$ Output of the ER cues model compared to human performance by vowel

consider a range of parameters to gauge the relative benefits of the two types of encoding.

\section{Exemplar model 2: manipulating free parameters}

In the next simulations with the exemplar model, we manipulated the $\tau$ and $r$ values in the similarity metric (using values of 1 or 2 for each) and the number of comparison tokens included in each exemplar cloud (including 10, 50, 200 or the full 330 tokens in each cloud). Fully crossing these parameters led to 16 different parameter settings. For each of these settings, we optimized the weights for the raw and ER models and then gauged categorization performance on the held-out tokens.

Overall, performance was quite comparable between the two classes of models (Table 2). Across both types of models, there was a slight overall advantage for models with $\tau$ and $r$ of 1 , which may suggest that the dimensions measured in this study should be treated as separable; however, given the similarly strong performance for models with $\tau$ and $r$ of 2, this conclusion is necessarily tentative. We also found that the $\tau$ and $r$ parameters interacted with each other (Fig. 2); when both were 1 or both were 2, both ER and raw cue models had a closer fit to human data than when they were unequal.

Moving to the relative advantage for ER and raw cue models, the average $L L$ across the different parameter settings (using the highest $L L$ across guessing rates for each model) showed an extremely small benefit for ER models $\left(L L_{\text {raw }}=-3,828 ; L L_{\mathrm{ER}}=-3,801\right)$. Of the 16 
Table 2 Model performance of the exemplar models as a function of the free parameters. For each parameter setting, the highest log likelihood (LL) is presented for the raw and expectation-relative (ER) models across all guessing rates

\begin{tabular}{|c|c|c|c|c|}
\hline$\tau$ & $r$ & Comparison tokens & $L L_{\text {raw }}$ & $L L_{\mathrm{ER}}$ \\
\hline \multirow[t]{8}{*}{1} & \multirow[t]{4}{*}{1} & 330 & $-2,239.36$ & $-2,211.14 *$ \\
\hline & & 200 & $-2,239.33$ & $-2,211.11^{*}$ \\
\hline & & 50 & $-2,239.78$ & $-2,214.65^{*}$ \\
\hline & & 10 & $-2,285.18$ & $-2,251.51 *$ \\
\hline & \multirow[t]{4}{*}{2} & 330 & $-3,859.14^{*}$ & $-4,197.42$ \\
\hline & & 200 & $-3,859.14^{*}$ & $-4,779.45$ \\
\hline & & 50 & $-3,859.14^{*}$ & $-5,039.22$ \\
\hline & & 10 & $-4,050.25^{*}$ & $-4,908.68$ \\
\hline \multirow[t]{8}{*}{2} & \multirow[t]{4}{*}{1} & 330 & $-6,255.2$ & $-5,197.88^{*}$ \\
\hline & & 200 & $-6,242.87$ & $-5,201.81^{*}$ \\
\hline & & 50 & $-6,407.28$ & $-5,474.01 *$ \\
\hline & & 10 & $-6,342.26$ & $-5,756.57^{*}$ \\
\hline & \multirow[t]{4}{*}{2} & 330 & $-2,838.46$ & $-2,829.86^{*}$ \\
\hline & & 200 & $-2,838.42$ & $-2,829.88^{*}$ \\
\hline & & 50 & $-2,838.95$ & $-2,832.73 *$ \\
\hline & & 10 & $-2,853.7 *$ & $-2,876.58$ \\
\hline
\end{tabular}

*Models with a better fit to the human perceptual data

parameter settings, 11 offered a closer fit to human data for the ER model, whereas 5 had a closer fit for the raw model. If we consider the 8 best fitting models in which $\tau$ and $r$ matched performance for ER and raw models was similar, the ER models were better in 7 of 8 .

The number of comparison tokens included in each fricative category also impacted the models' fit to human data (Fig. 3). For both raw and ER models, fit was better for models that included a larger number of comparison tokens. When only a small number of tokens were in the comparison set (10 or 50 tokens), the raw models offered a closer fit to the human data than did the ER models. This benefit was reversed when including larger comparison sets; the largest difference

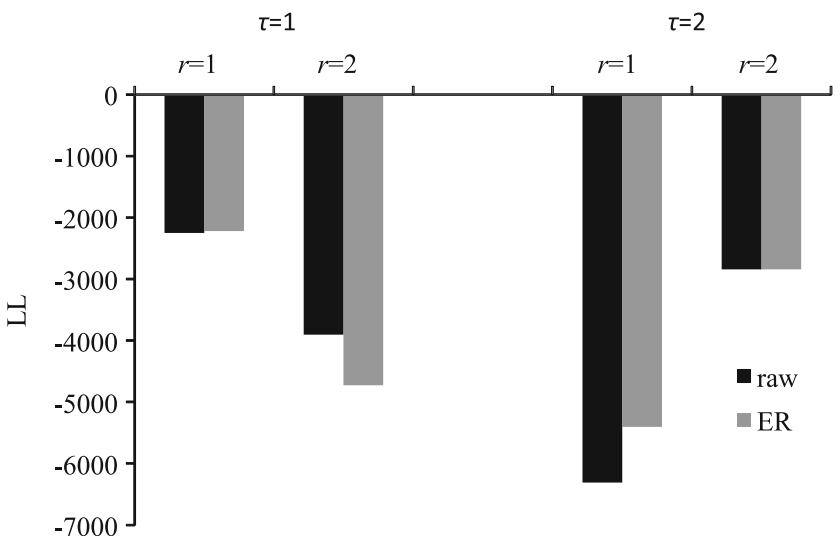

Fig. 2 Log-likelihood (LL) for models with different values of $\tau$ and $r$, averaged across the different number of comparison tokens

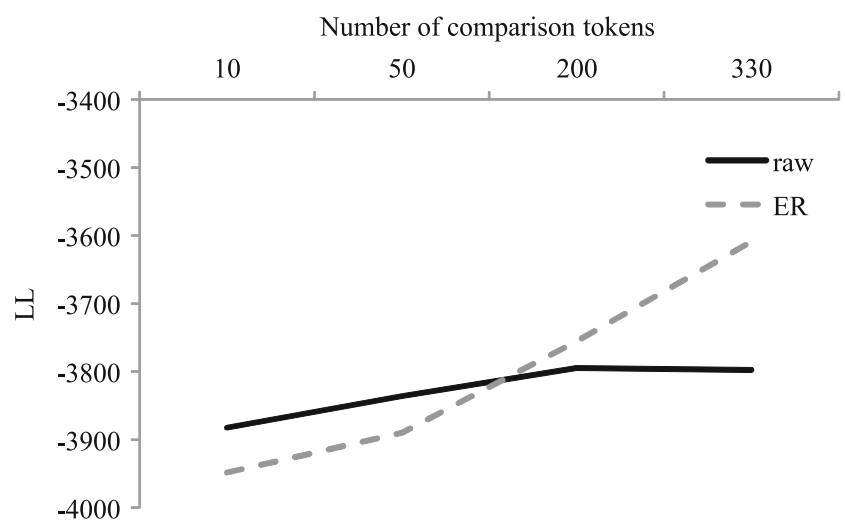

Fig. 3 LL as a function of number of comparison tokens in each fricative category and model class, averaged across $\tau$ and $r$

between the model types was with the full set of 330 comparison tokens (averaged across the other parameters: $L L_{\text {raw }}=-$ 3,798; $\left.L L_{\mathrm{ER}}=-3,609\right)$. This increasing benefit for ER models with more comparison tokens suggests that scaling the model up toward the number of exemplars a real listener encounters may maintain the benefit for ER encoding.

Finally, we can consider the best parameter setting for each class of model to determine which offered the closest fit overall to the human data (Table 3). For both models, the best-fitting model had a $\tau$ of 1 , an $r$ of 1 and 200 comparison tokens. These models showed a very small benefit for ER encoding $\left(L L_{\text {raw }}=-2,239 ; L L_{\mathrm{ER}}=-2,211\right)$.

These simulations show a mix of evidence for raw and ER encoding depending on model parameters, with a very slight overall benefit for ER encoding. These results do not support an overwhelming benefit for ER encoding, suggesting that exemplar models may accommodate talker and context variability without the need for computing cues relative to expectations. Thus, relative cue encoding may not be the only way to effectively solve the lack of invariance problem; using this classic form of exemplar model, raw cue encoding appears to

Table 3 Results from parameter searches in different model architectures, sorted by model type. Max values are taken from the simulation in that parameter search which gave the high values. The percent better fit signifies how many models in that class elicited a closer fit to the human data, across parameter and guessing rate settings. $P D P$ Parallel distributed processing

\begin{tabular}{|c|c|c|c|}
\hline Simulation & Model Type & $\operatorname{Max} L L$ & Percent better fit \\
\hline \multirow[t]{2}{*}{ Exemplars: Include talker } & Raw & $-2,239$ & $31.2 \%$ \\
\hline & ER & $-2,211$ & $68.8 \%$ \\
\hline \multirow[t]{2}{*}{ Exemplars: without talker ${ }^{a}$} & Raw & $-5,061$ & \\
\hline & ER & $-3,692$ & \\
\hline \multirow[t]{2}{*}{ PDP } & Raw & $-2,506$ & $5.6 \%$ \\
\hline & ER & $-2,358$ & $94.4 \%$ \\
\hline
\end{tabular}

a Only one exemplar model excluding talker was run for each class, so the percentage of better fitting models is not provided 
result in performance that is approximately as good as ER encoding.

However, a potential concern about the benefit for raw cue encoding is that it may have benefited primarily from nearidentical tokens in the training set. Repetitions of the same fricative by the same talker in the same vowel context were included in the training and testing sets; a raw exemplar model can use these extremely close tokens as the basis for categorization. For the ER models, meanwhile, removing context from these exemplars may ensure that many tokens are reasonably close to the stimulus, but it may also eliminate indexical variability, making the closest stimuli slightly poorer matches. The strong performance of the raw models may thus derive from similarity to such extremely similar tokens; this is particularly worrisome as the raw models were particularly effective when using a small comparison set. This raises questions as to how well a raw model could generalize to new talkers.

Repeated tokens of the same speech segments by the same talkers are frequently encountered by listeners, so perhaps this matching to exact repetitions is not problematic. However, listeners in the behavioral study conducted to collect the perceptual data used to assess model performance (McMurray \& Jongman, 2011) had no experience with the talkers before beginning the experiment; instead, they had to perform identifications by generalizing from previous experience with other talkers to the new talkers encountered in the experiment. As such, we investigated whether the benefit for raw cue encoding is contingent on matching to tokens from the same talker, or if this advantage generalizes to when categorizing tokens from novel talkers.

\section{Exemplar model 3: generalizing to new talkers}

To simulate identification for novel talkers, we retested the model, this time comparing the incoming token to those exemplars in the training set from the other 19 talkers, but excluding those exemplars spoken by the talker of the test token. To this end, we retrained the models to produce individual weight matrices excluding each talker from the testing set. Thus each testing token was considered given a model that had never been exposed to tokens from that talker. This reduced the training set from 2,640 tokens to 2,520 tokens for each test talker (the exact set of 2,520 tokens varied depending on which talker was used). We assessed performance on a model with a $\tau$ of 2 , an $r$ of 2 and 10 comparison tokens from each fricative category; this parameter setting was the best-fitting model for which the raw model outperformed the ER model. Additionally, assessing a model with this small subset of tokens provides a stringent test of whether extremely nearby tokens from the same talker are driving performance for the raw model. We thus calculated ten sets of weights for both the raw and ER models (one set of weight excluding each of the ten talkers included in the testing set). These weights were computed exactly as the previous models, just using a slightly different subset of tokens each time. ${ }^{7}$ At test, we used these weights to calculate performance for the 240 test tokens, again excluding the talker of each test token from the comparison set.

Both the raw and ER model had a substantially poorer fit to the human data with this adjustment (Fig. 4). However, the decrement in fit was much more pronounced for the raw models $\left(L L_{\mathrm{raw}}=-5,061 ; L L_{\mathrm{ER}}=-3,692\right.$; when including the same talker in the comparison set, these values were $-2,854$ and $-2,877$, respectively). Both models predict performance below that attested by humans for most fricatives; the raw model is particularly poor at predicted performance for the sibilants. This suggests that matching exemplars from the same talker was beneficial to both classes of models, as performance declined for both; however, the raw models were relying much more heavily on these nearby tokens. Without access to similar tokens, the raw models struggled quite severely, suggesting impaired generalization.

These simulations of generalization offer a more direct analog of the perceptual study used to collect the human comparison data. Those participants were identifying tokens from talkers with whom they had no prior experience. As such, the participants were forced to rely on previous tokens from other talkers to make their identifications. These results also suggest that the previous high performance for raw cue models was a result of matching to near-identical tokens from the same talker. This strategy was not available to listeners in the perceptual study, who were unfamiliar with the talkers. However, it is possible that in more familiar listening situations, listeners could adjust their identification strategies to make better use of this direct-exemplar matching ability. When listening to a highly familiar talker speaking in a familiar context, listeners may not recode the incoming stimuli, but could instead compare directly against stored exemplars from this same talker. However, they could achieve a similar benefit by recoding cues relative to the expected cuevalues for that speaker. Moreover, when encountering a new listening situation (e.g., a new talker, a room with different acoustics, a changed speaking rate), recoding likely proves more useful.

\section{Exemplar models: discussion}

Across a range of exemplar models, we found very limited support for raw cue models. When models had a nearly exact

\footnotetext{
${ }^{7}$ Unsurprisingly, the weights changed very little between the different subsets; as 18 of the 19 talkers were shared between any two models, the training sets had extremely high overlap.
} 

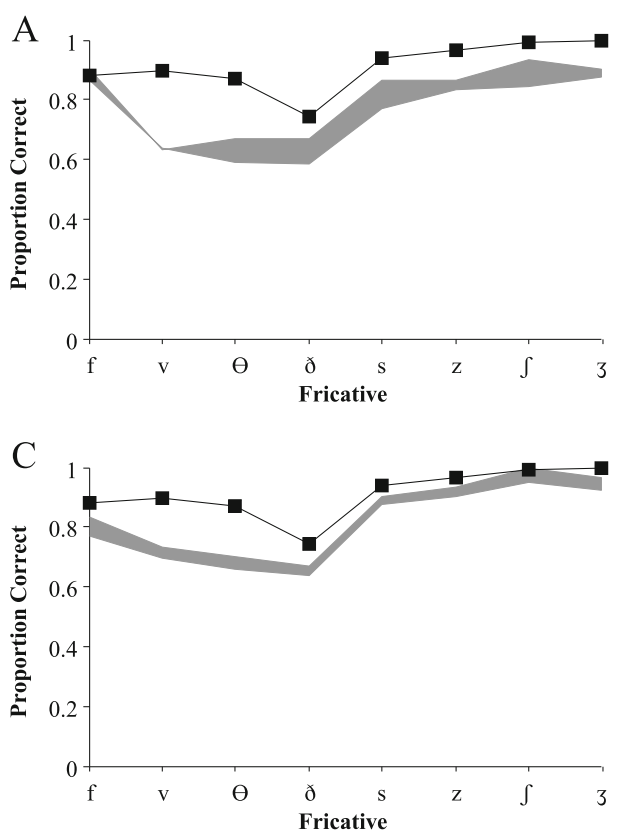

Fig. 4 a-d Model outputs compared to human data for the talkerexcluded models. a Output of the raw cues model compared to human performance by fricative. b Output of the raw cues model compared to

match to the input in the exemplar store, raw models were approximately as effective as ER models, and they showed a slight advantage with comparison sets that included only a small number of exemplars. More importantly, when tested on generalization to novel talkers, ER models showed a distinct advantage.

It is important to note that even in the talkerexcluded condition, valuable surface variability was available to aid exemplar categorization using raw cues. All of the fricatives had exemplars with all six different coarticulatory contexts, and there were nine other talkers of the same gender. These are the kind of contexts that should have promoted flexible generalization. Yet even with these advantages, the raw cue models fared worse than ER models, suggesting that the ability of exemplar models to overcome this kind of variation may only extend to situations in which nearly exact matches can be found in the exemplar cloud.

Exemplar models thus do not appear to obviate the need for some form of acoustic recoding prior to categorization, at least when encountering novel talkers. Despite their power in dealing with variability by harnessing context-specific information, raw acoustic information does not result in better performance, and in typical cases ER encoding shows a benefit. Such ER benefits likely emerge from the large variety of contexts from which our training exemplars come, which appear to overwhelm raw models, but which provide excellent fodder for forming precise expectations (and which offer a closer approximation to human speech perception experience). The huge
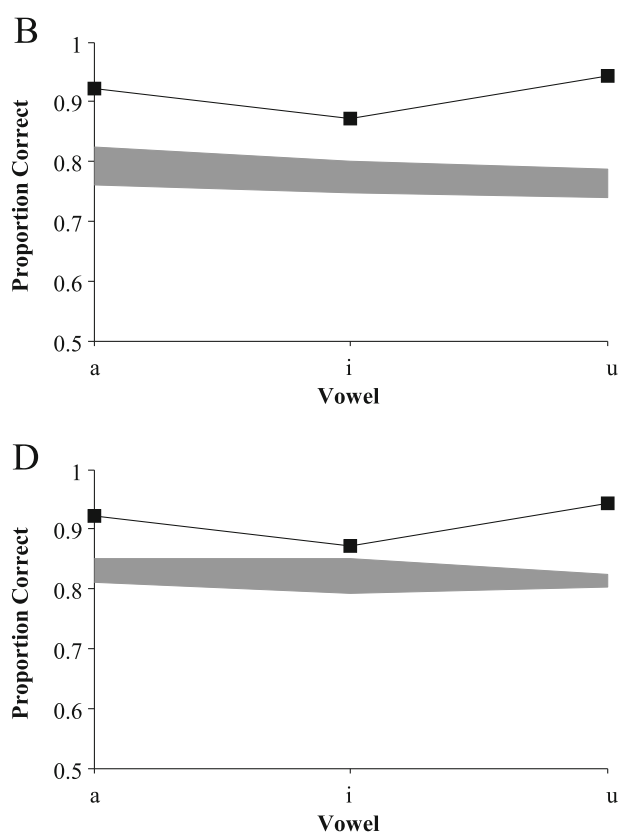

human performance by vowel. $\mathbf{c}$ Output of the ER cues model compared to human performance by fricative. d Output of the ER cues model compared to human performance by vowel

range of variability, coupled with the complex set of cues useful for fricative identity, means that even exemplar models need some external scaffolding to help deal with contextual variability to demonstrate human-like phoneme recognition. If listeners use exemplar systems to recognize speech sounds, they might not do so using raw acoustic information, at least when hearing new talkers or in novel contexts.

Although exemplar models do not benefit from raw acoustic encoding, it is unclear whether human speech perception uses such exemplar processes to perform phoneme identification. Indeed, one might argue that if stimulus recoding is necessary, there is little additional benefit for exemplar models. Although these models were somewhat accurate in fitting the perceptual data, the logistic models analyzed by McMurray and Jongman (2011) also offered close fits (their best-fitting model had a $L L$ of 2,398 , compared to the best-fitting exemplar model's $L L$ of $-2,211$; the exemplar model was a better fit, although not overwhelmingly so). These specific results do not definitively favor either architecture as a better model of human processing, although they encourage further investigation of exemplar models in this domain. However, both cases showed support for the use of ER cues by listeners. Indeed, some evidence for talker specific effects may be accounted for by simply associating talker voice with word-forms (Creel et al., 2008) or even by talkercontingent recoding of cues (e.g., Nygaard, Sommers, \& Pisoni, 1994), which do not require an exemplar architecture. 


\section{PDP models}

In PDP models, information is processed through a hidden layer (or series of hidden layers) based on learned connection weights with other layers (Rumelhart et al., 1986). Using one or a series of hidden layers between the input and output layers, these models can learn subtle patterns in the input as well as higher order correlations between inputs. The activation dynamics defining how activation flows to these layers allows PDP models to approximate prototype models, extracting (often difficult) category structure information.

The back-propagation learning algorithm, which uses the error between performance and a teaching signal to alter connection weights, allows a model to learn input patterns that are unapparent with simpler learning mechanisms. It can separate classes of inputs without linear boundaries. Especially when many hidden units are available, PDP networks can make use of idiosyncratic or context-specific aspects of the signal, similar to exemplar models, allowing them to function as a type of hybrid between prototype and exemplar categorization systems. Thus PDP networks are a valuable place to explore informational assumptions in speech perception; if, as proposed by advocates of raw acoustic phoneme perception, expectation relative encoding is unnecessary, then altering the stimuli by removing effects of talker and phonetic context may lead to poorer fits to human patterns of identification.

Using the same set of raw and ER cue values, we gauged the ability of a range of PDP models to demonstrate fricative identification abilities. As before, all models were trained with 2,640 items from the eight fricative categories, and then were tested on 240 held out tokens. Each model was tested using both raw cues and ER cues to determine how relative cue encoding affects model performance, and how this compares to human performance.

\section{Implementation}

\section{Base architecture}

Our base PDP model consisted of a bank of input units representing the 24 acoustic cues; a bank of hidden units receiving connections from the input units and sending connections to the output units; a bank of output units representing the eight fricative categorization choices; and bias weights affecting the output units (Fig. 5).

The activations for input units were continuous and set to the cue values from the corresponding stimulus set (either raw or ER). Hidden units used the typical logistic activation function (with a temperature parameter; this was not trained, but was manipulated as a free parameter) to convert activation from the inputs into a squashed value between 0 and 1 . The output layer consisted of eight units representing the eight

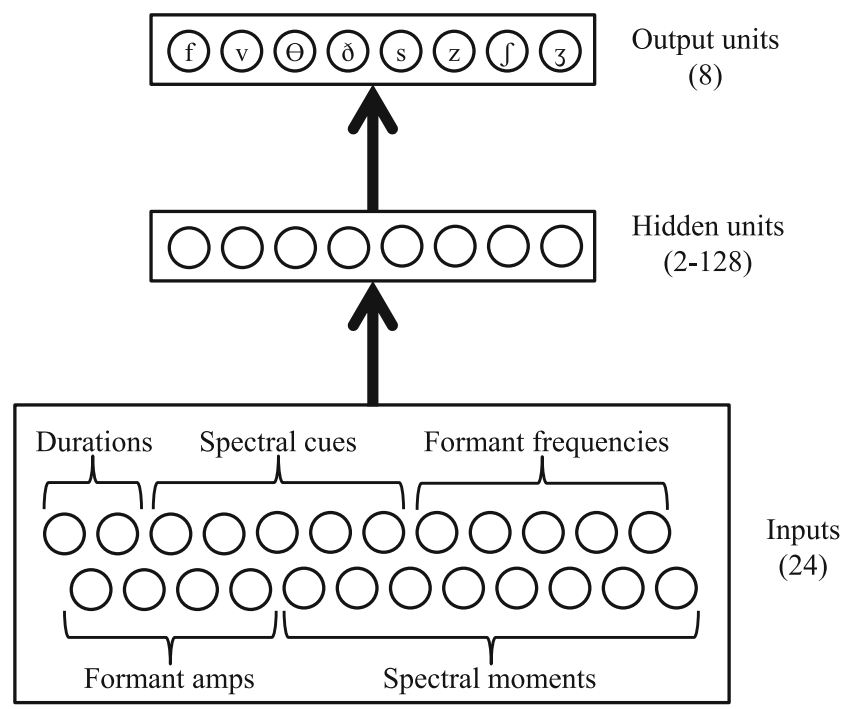

Fig. 5 Architecture of the parallel distributed processing (PDP) network. The number of units in a layer is in parentheses. The number of hidden units was a free parameter

English fricative categories. These units received activation from the hidden layer and a learnable bias weight, and transformed this to probabilities of a given response using the softmax function. All weights and bias weights began with random values between 0 and 0.1 .

\section{Training}

Weights were trained with the standard back-propagation algorithm. The error signal at the output layer was computed as the difference between the target vector and the actual output; the target vector was a vector in which the correct (produced) fricative unit had an activation of 1 and all other units had an activation of 0 . There were two free parameters: the learning rate, which controls how quickly the weights should change on any epoch; and the momentum, or the amount of carryover learning from previous trials. Each model was trained by randomly choosing one of the 2,640 training exemplars, calculating output activation, and then calculating error and adjusting weights. This continued for as many trials as were chosen to train that model (see Parameter space, below). For testing, each held-out stimulus was presented to the model once, and no learning occurred.

\section{Testing the model}

As in the exemplar models, we present accuracy from both the discrete choice and probabilistic linking functions. Quantitative analysis of these models was accomplished using $L L$ calculations as well as the guessing rate adjustments used in the exemplars models. 


\section{Parameter space}

PDP models offer a wide range of different parameter settings. While we do not attempt to complete an exhaustive analysis of all possible parameters, we adjusted several to determine if any parameter settings affect the benefits or detriments resultant from using ER inputs, and to determine how consistent benefits for either class of model are across parameter settings (Pitt et al., 2006).

The manipulated parameters (Table 4) included (1) the number of training epochs before testing; (2) the learning rate; (3) the strength of momentum during learning; (4) the number of hidden units; and (5) the temperature of the logistic activation function. The number of hidden units was of particular interest, as this parameter affects how a model encodes information: fewer hidden units forces greater abstraction. We predicted that greater numbers of hidden units, with their ability to abstract as well as encode context-specific information, would offer the best environment for raw cue models, while fewer hidden units would promote ER cues. Given the number of parameters, there were too many models to analyze individually. Instead, we looked more holistically at the results to determine how often raw cue models outperformed ER models and vice versa. Where more useful, we present specific, meaningful simulations demonstrating interactions of specific parameters. This form of analysis allows us to determine whether our pattern of results is dependent on specific settings of the number of free parameters, or if instead many of these parameters have little effect on the findings of interest.

\section{Results}

On the whole, models using ER cue values outperformed those using raw values, although the mean performance was unimpressive. Across all parameter settings, the ER models were more accurate than the raw cue models (ER models: 51.3-63.4 \% correct; raw cue models: 48.2-59.4 \%). Similarly, the ER models

Table 4 Free parameters and investigated values for single-hiddenlayer and multiple-hidden-layer PDP models

\begin{tabular}{ll}
\hline Free parameter & Investigated values \\
\hline $\begin{array}{l}\text { Number of hidden units } \\
\text { per hidden layer }\end{array}$ & $2,4,8,16,32,64,128$ \\
$\begin{array}{l}\text { Learning rate } \\
\text { Temperature (logistic } \\
\text { activation function) }\end{array}$ & $0.0001,0.001,0.01,0.05$ \\
$\begin{array}{l}\text { Momentum in learning } \\
\text { Number of training } \\
\text { epochs }(\times 100,000)\end{array}$ & $0.25,1,2,4,8$ \\
\begin{tabular}{l} 
Guessing rate \\
\hline
\end{tabular} & 1 hidden layer: $1,2,3,4,5$ \\
\hline
\end{tabular}

offered a closer fit to the human data (averaged across all guessing rates: $\left.L L_{\mathrm{ER}}=-9,018 ; L L_{\text {raw }}=-9,597\right)$.

Overall, these models were far less accurate than the exemplar models presented here and the logistic models of McMurray and Jongman (2011). However, this decrement in accuracy reflects a wide range of performance across a very large parameter space; some parameter sets led to very poor learning, whereas others offered quite impressive performance, with maximum performance of 89.2-94.2\% correct (Table 3), which encompasses human performance levels $(91.2 \%)$. Similarly, some models had fits to human data that were in a similar range to the fits seen in the exemplar models and the McMurray and Jongman (2011) models (max $L L=$ 2,358). As models with extremely poor performance are poor approximations of human processing, we considered only cases where the parameter setting was appropriate for at least one type of model (raw or ER) to yield reasonable accuracy. This was set at $80 \%$ by the discrete rule - approximating the simplest raw cue model from McMurray and Jongman (2011) using the same dataset. Of all parameter settings, $49 \%$ were excluded, including all models using only two hidden units. Including all parameter-sets in our analysis yielded qualitatively similar results with respect to benefits for ER encoding.

Considering only the successful models, the ER models were about $4 \%$ more accurate than the raw cue models (ER: 79.4-88.5\%; raw: 74.6-84.5\%; Table 3). More importantly, ER models also fit the perceptual data considerably better than raw cue models (averaged across models and guessing rates; $\left.L L_{\mathrm{ER}}=-3,721 ; L L_{\text {raw }}=-4,365\right)$; this benefit was also quite consistent: $94.4 \%$ of parameter settings exhibited a better fit when using ER cues.

We next examined the most effective models of both types. First, we identified the models with the highest accuracy. The best raw cue model (Fig. 6a,b) correctly identified 84.8$92.9 \%$ of tokens $\left(L L^{8}=-2,776\right)$, whereas the best ER model (Fig. 6c,d) was 89.2-94.2\% correct $(L L=-2,400)$; the most accurate ER model was both more accurate and, more importantly, a better fit to human performance, than the raw model. Although both classes of models did a fair job matching the qualitative pattern of accuracy across different fricatives, contrary to human performance the raw cue model performed best for fricatives that occurred in the context of the vowel /i/ (Fig. 6b). The ER model more closely mirrored human performance, showing the expected decrement in identification of fricatives in the context of /i/ (Fig. 6d). This better qualitative fit is reflected by the improved quantitative fit seen in $L L$ values. Interestingly, despite our predictions that the number of hidden units would be a strong determiner of whether raw or ER models performed better, the most accurate models in

\footnotetext{
${ }^{8}$ These $L L$ values are the max $L L$ s for each model across all guessing rates. For both models, this occurred at guessing rate $=0.1$.
} 

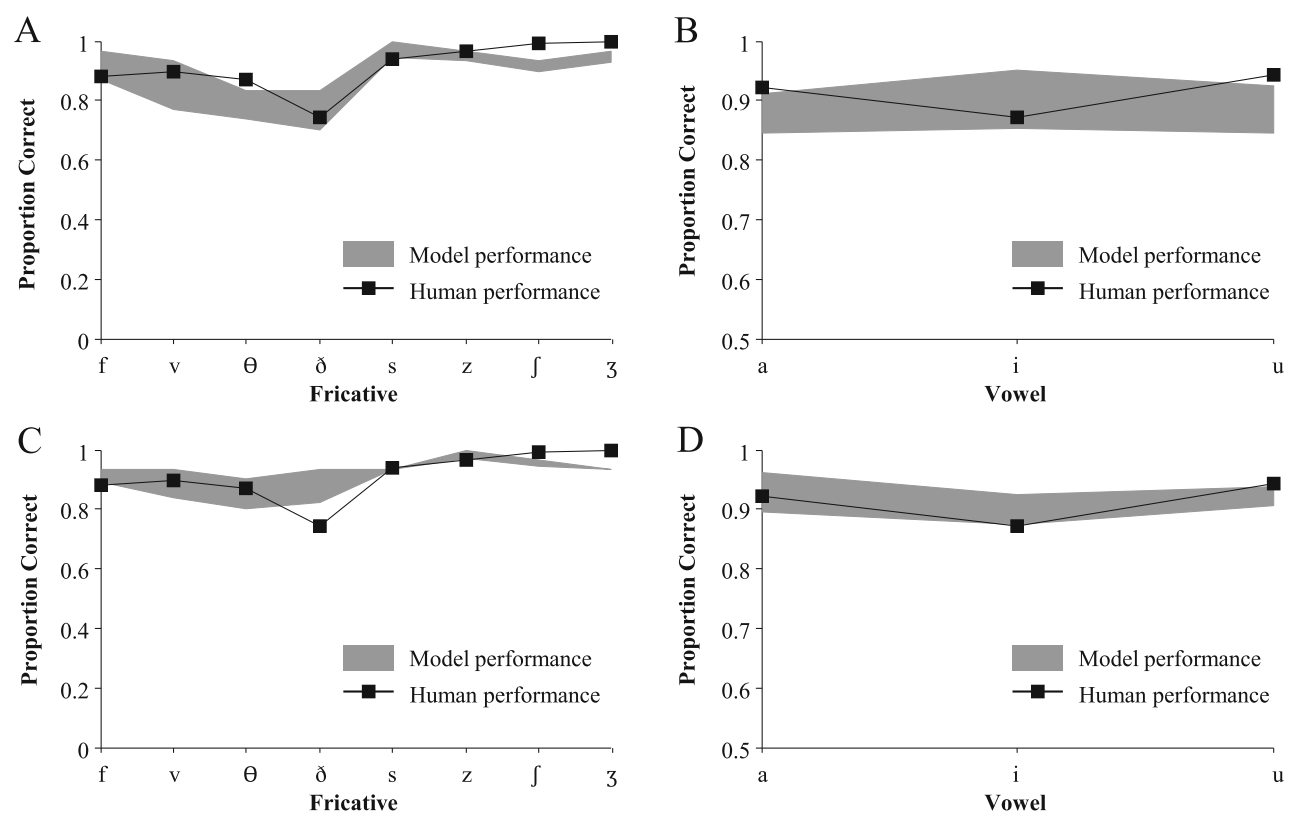

Fig. 6 a-d Performance for the most accurate PDP models. a Raw cue model by fricative. b Raw cue model by vowel. c ER cue model by fricative. d ER cue model by vowel

both cases had 32 hidden units (other models included as many as 128 hidden units).

We next examined models with the closest fit to the perceptual data. These models were not the same models as the most accurate; as with the exemplar models, models with high accuracy did not necessarily excel on the same tokens as human listeners - accurate models are not necessarily ones that mimic human performance. We considered all models at all guessing rates to determine which models exhibited the highest $L L \mathrm{~s}$. The best fitting raw cue model had a $L L$ of 2,506; the best ER cue model gave a better fit $(L L=-2,358)$. These models are shown in Fig. 7. As in the most accurate models, model performance in different vowel contexts proves quite diagnostic; the ER model correctly predicts worse performance on /i/ (Fig. 7b), whereas the raw cue model does not (Fig. 7d). For each of these models, we also examined the corresponding parameter setting for the opposite model type. In each case, the other model type showed a worse fit; for the top raw cue model, the corresponding ER model had a very slightly worse fit $\left(L L=-2,558 ; \Delta_{\mathrm{LL}}=52\right)$. The top ER model was paired with a raw cue model with a much poorer fit $\left(L L=-2,980 ; \Delta_{\mathrm{LL}}=622\right)$.

Each class (raw vs. ER) of model showed peak performance that surpassed that of the other class with the same parameter set (although for the peak raw cue model, this benefit was small). That is, the best ER model was better than the equivalent raw cue model, but the best raw-cue model was also better than the equivalent ER model. To ensure that ER benefits were not a result of cherry-picking from beneficial regions of parameter space, and to determine whether any of the free parameters drove this advantage, we next examined the full range of parameter settings to determine the relative benefit of raw and ER input. This analysis comprised 1,782 models of each type (those models surpassing the accuracy cutoff and for which both models yielded real number $\left.L L s^{9}\right)$, analyzed at seven guessing rates.

Figure 8 shows the results from this analysis. The bulk of parameter settings exhibited greater $L L$ values for ER than for raw-cue models ( $94.4 \%$ of cases). This was fairly consistent across all guessing rates $(\min =93.0 \%, \max =98.4 \%)$. Analyzing only the best guessing rate for each model (that is, for a given parameter setting, the guessing rate that yielded the highest $L L$ for each model type), again showed dominance for ER cue models; for $96.3 \%$ of parameter settings, the best ER model had a higher $L L$ than the best raw cue model. The ER benefits were quite independent of parameter settings, and were extremely consistent.

\section{Hidden units}

Overall, ER models outperformed raw cue models for the vast majority of parameter sets. However, we suspected that at least for some classes of PDP models (those with many hidden units), ER encoding may be unnecessary as these models can both abstract from the acoustic signal and encode exemplarspecific information. Thus, we specifically analyzed performance and fit as a function of the number of hidden units.

\footnotetext{
${ }^{9}$ For one parameter setting, the raw model did not elicit real number $L L \mathrm{~s}$ at any guessing rate. All other models that surpassed the accuracy threshold had viable $L L$ s for both types of model.
} 

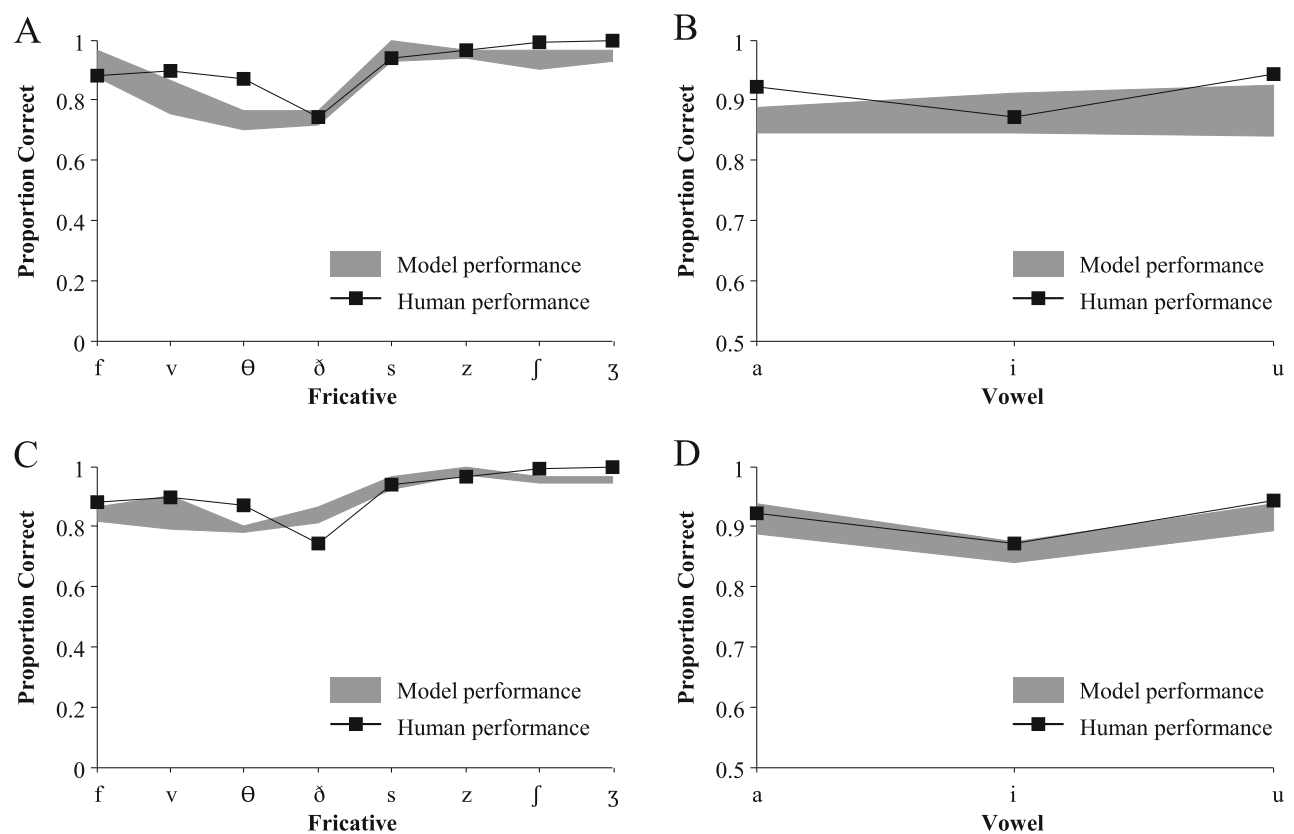

Fig. 7 a-d Model performance for PDP models with the highest LL values. a Raw cue model by fricative. b Raw cue model by vowel. $\mathbf{c}$ ER cue model by fricative. $\mathbf{d}$ ER cue model by vowel

Despite these predictions, we found a benefit for ER encoding at all numbers of hidden units (Fig. 9). For both classes of models, performance was only moderately affected by the number of hidden units; with greater than four hidden units, accuracy was approximately the same. However, when measured by $L L$, model fit decreased as the number of hidden units increased. More interestingly, as the number of hidden units increased, the benefit for ER cues also increased. ${ }^{10}$ With more hidden units, the raw cue models surprisingly fell farther behind the ER models: in parameter-sets where the model was maximally designed to harness exemplar-specific information, the benefit for ER encoding was the strongest.

\section{Other factors}

Although the number of hidden units was expected to be the factor most likely to affect the relative benefits of ER encoding, other parameters may have influenced performance in less predictable ways. To get a clearer picture of how the various other parameters interacted to exhibit the different behaviors of the models, we performed a linear regression analysis of model performance. Our DV was the difference in $L L$ between the raw and ER models (which is essentially a log odds ratio), and the free parameters (number of hidden units, learning rate, temperature, momentum, number of epochs and guessing rate) served as the predictors. Only parameter

\footnotetext{
${ }^{10}$ When we included all models instead of just those with accuracy above $80 \%, L L$ measures decreased with more hidden units, suggesting greater incidence of poor learning with more hidden units. However, the larger benefit for ER models in cases with more hidden units also held in this analysis.
}

settings that surpassed the accuracy threshold and yielded real number $L L$ s with both raw and ER inputs were included in the regression. For this analysis, we divided the number of training epochs by 10,000 to make the $B$ coefficient more interpretable.

Several free parameters affected the degree of benefit for ER cues. Confirming the analysis above, as the number of hidden units increased, the benefit for ER cues grew $(B=3.16$, $\left.S E=.12, R^{2}=.051, P<.0005\right)$. Similarly, the benefit for ER models was greater at higher temperatures $(B=21.84, S E=$ $\left.1.92, R^{2}=.010, P<.0005\right)$ and with more training epochs $(B=$ $\left.2.94, S E=.39, R^{2}=.0045, P<.0005\right)$. The benefit for $\mathrm{ER}$ was larger for lower learning rates $(B=-1,369.28, S E=255.59$, $\left.R^{2}=.0023, P<.0005\right)$. Momentum $\left(R^{2}<.0001\right)$ and guessing rate $\left(R^{2}<.0001\right)$ did not affect degree of ER benefit.

The benefit for ER cues varied across different parameter settings. Two aspects of this variability are particularly noteworthy. First, as discussed above, increasing the number of hidden units led to greater advantage for ER models, suggesting that models that potentially have more opportunity to encode exemplar-specific information are nonetheless improved by using cues that are relative to expectations. Second, the effect of training epochs showed a greater advantage for ER cues after more training. This suggests that, as learning continues, the models come to depend less on stimulus-specific information and more on relative cue encoding. Although various parameters interacted with the degree of ER benefit, no settings of individual parameters consistently showed a benefit for raw encoding; ER benefits thus do not appear to result from cherry-picking fortuitous regions of parameter space. 


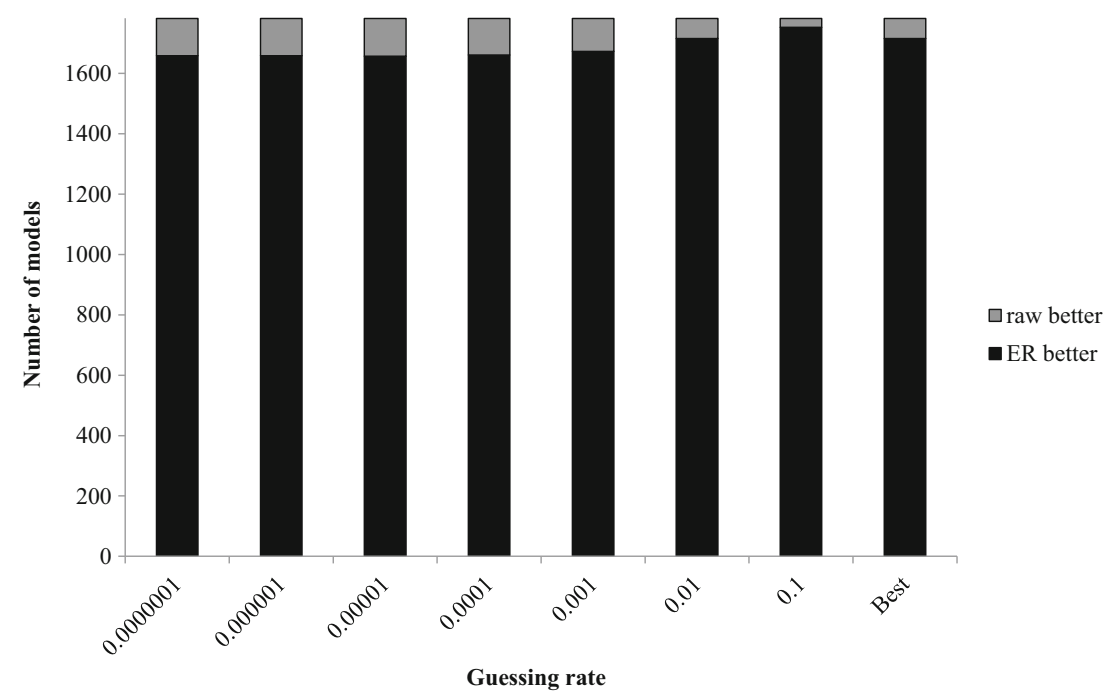

Fig. 8 Frequency of benefit for each class of model at different guessing rates

PDP models: discussion

Like exemplar models, PDP models offered a promise of accounting for variability in the speech signal without the need for ER encoding. With the ability to both abstract away from variations and encode stimulus-specific information to deal with context effects, these models seemed poised to process difficult speech stimuli using only raw cues. However, despite the fact that many of these models showed excellent categorization, in the vast majority of circumstances PDP models were more successful with ER cues as input. This benefit was nearly constant: over $94 \%$ of parameter settings gave a closer fit to human perceptual data when using ER cues. While a handful of settings yielded better performance for raw cues, these models tended to offer only a modest benefit over the ER cue models.

We predicted that models with a larger number of hidden units would allow better performance for the raw models, as this expanded hidden unit space could provide a means for raw models to make the most out of both exemplar-specific and abstract encoding. However, the opposite was found: models with more hidden units showed an increased advantage for ER encoding. The unexpected result may arise because raw models with a large hidden unit space have more capacity to encode

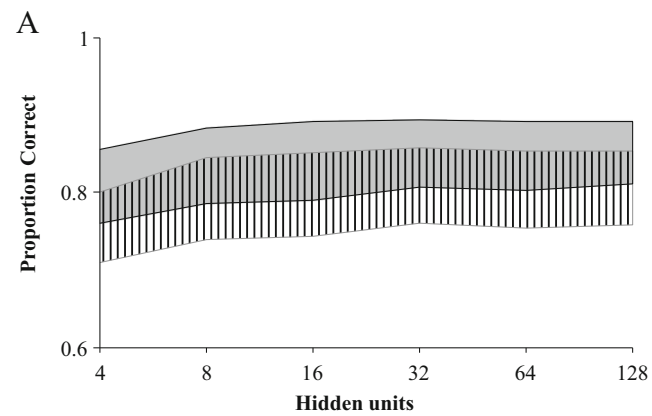

idiosyncratic aspects of the training tokens without abstraction. That is, with more hidden units, models have less need to perform dimension reduction on the inputs. Including fewer hidden units may thus have forced the raw cue models to attempt to better account for context (although these models still did not accomplish this as well as the ER models).

These analyses of PDP models of fricative perception thus show a fairly clear advantage for information that is encoded relative to expectations. Despite the possibility that PDP models had sufficient computational power to overcome variability in the signal without recoding input, or to perform a form of relative encoding on their own, or to find an invariant cue or combinations of cues in the raw signal, ER encoded cues resulted in consistently better model performance. The choice of information content thus had major ramifications on model categorization performance.

\section{General discussion}

We investigated how the encoding of information affects categorization performance in different architectures.

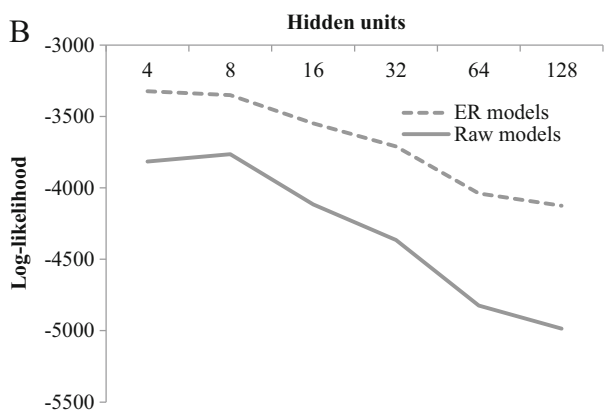

Fig. 9 a Percent correct of raw and ER cue models at different numbers of hidden units. b LL of the perceptual data given different model types and different numbers of hidden units 
Whereas previous research on categorization has focused heavily on the architecture of categorization independent of informational assumptions, we investigated the impact of these informational assumptions along with architecture. To this end, we focused on a domain in which the way information is encoded is a matter of debate - speech perception - as here categories are delineated less clearly than in many laboratory studies of categorization, and there are debates about the nature of the input supporting categorization.

We investigated categorization performance for a large corpus of acoustic measurements of speech tokens. Previous investigations using this same dataset found improved fits to human perceptual performance when using ER cues (the basis of predictive coding schemes) compared to using raw cues (McMurray \& Jongman, 2011). However, this previous work used only a single, simple categorization architecture that may have offered an unfair advantage for the re-coded speech information; their prototype-style categorization architecture has no other mechanisms with which to cope with this variability. We investigated other categorization architectures: exemplar and PDP models. Both of these systems offered the promise of accounting for variability as part of the categorization architecture, suggesting that re-coding schemes that remove such variability might impair the capacity of the models to mimic human categorization.

Despite this possibility, across many parameter-sets, ER cues consistently elicited performance that closely resembled human performance from both architectures, whereas raw cue models lagged behind in many situations. This improvement occurred despite exemplar models' explicit reliance on holistic matching to surface-form variability, and held even for the subset of PDP models whose architecture offered the most potential for embracing surface form variability (those with many hidden units). In both classes of models, the best-fitting models overall showed a benefit for ER encoding in terms of fit to human data (although this benefit was modest for exemplar models that included the test talker in the comparison set). The exemplar models also showed clearly that ER encoding better accounts for generalization abilities than does raw cue encoding. In short, ER encoding offered the closest fit to human categorization, independent of model architecture, suggesting that listeners utilize such a mechanism.

Overall, the exemplar models that best matched human performance $(L L=-2,211)$ offered a closer fit to human performance than the top PDP models $(-2,358)$ and McMurray and Jongman's (2011) C-CuRE model $(-2,398)$. This benefit was fairly modest, however, and was attested in those exemplar models that were benefitting particularly from comparisons to tokens from the same talker. The PDP models and the C-CuRE model yielded extremely comparable performance. This may seem to suggest that exemplar models provide the best account for fricative perception in humans, but such a conclusion seems highly dependent on whether or not the listener is presumed to have knowledge of the talker, and may depend on whether we presume ER or raw encoding in the exemplar models.

Additionally, the exemplar models, when including the test talker in the comparison set, showed nearly identical fits to human data for raw and ER models. Although an ER benefit was apparent when excluding tokens from the matching talker, the similar levels of performance by the two classes of models at their peaks diverged from the results of the PDP models and the McMurray and Jongman models, in which the ER models vastly outperformed the raw models. This pattern may suggest a non-trivial interaction between model architecture and the form of encoding; future work is needed to flesh out the nature of this interaction.

However, thorough comparison between the models is difficult as they used vastly different parameter spaces, and they were not proposed as comprehensive models of speech perception. Additionally, their similar overall performance resonates with recent proposals that comparisons between overall architectures of complex models is often fruitless, as they are often equally capable of simulating the same data under the right parameter settings (Yu \& Smith, 2012). The overall benefit for ER encoding across models should thus be seen as the more enduring finding from these simulations.

So, why does raw cue encoding hamper model performance for these architectures that would seem to be well equipped to deal well with surface variability? One likely source of difficulty for such models is that tokens from other contexts interfere with classification. For example, an $/ / /$ spoken by a female is acoustically quite similar to an /s/ spoken by a male. When classifying a male $/ \mathrm{s} /$, a raw cue model encounters other nearby $/ \mathrm{s} /$ tokens, but also nearby female $/ \mathrm{g} /$ tokens as well. This overlap decreases the model's ability to adequately classify the token. Meanwhile, an ER model accounts for this overlap; rather than storing information based on their raw acoustic similarity, tokens are stored relative to expectations: the male /s/ is encoded as having a high spectral mean relative to typical male productions. This allows ER models to circumvent difficulties that arise from acoustic overlap.

Superficially, our basic result may appear trivial: of course removing noise from the signal results in better performance. However, our findings of the type of noise reduction have major ramifications for understanding of speech perception. Many of the most heated debates in speech perception concern whether there is too much noise in the signal for various architectures to do the job (Fowler, 1986; Lindblom, 1996; Ohala, 1996). Here we've shown that accounting for a few sources of this noise with a very simple approach elicits performance very similar to human listeners, and furthermore shown that this so-called noise reduction is more important than the architecture of categorization for reaching these performance levels, as three distinct architectures all showed impressive fit to human performance. Moreover, the form of 
noise reduction used for these models was not optimized for the target problem (fricative categorization), but was instead a rather generic form of noise reduction. Thus, in the context of decades of work on speech perception, this finding is quite far from trivial.

Further, although the idea that removing noise should improve model performance is intuitive, it is less straightforward to determine whether such improved performance matches that of humans. The most accurate model did not always offer the best account of human performance. Thus the expected gains in model ability to correctly identify the speech segments when context is factored out do not imply that such models will necessarily mirror patterns of human performance.

The metaphor with noise reduction is an apt description of what expectation-relative or predictive coding schemes do: the expectations formed from other decisions about the speech signal help identify the source of types of variance, creating a more invariant signal for categorization. Whereas predictivecoding is generally implemented in complex architectures (often generative Bayesian frameworks), the demonstration here that similar functionality can be achieved with something as simple as linear regression speaks to the power of this broader principle, but also unifies work in speech perception with other domains in which predictive coding has been invoked. Like predictive coding accounts of other cognitive processes, contingent encoding accounts in speech perception remain controversial, with many researchers insisting that the raw acoustic signal is used for phoneme identification. Despite the intuitive nature of the idea that noise reduction can improve performance, in both predictive coding and contingent speech perception, there has remained considerable doubt about whether humans use this form of noise reduction. The degree to which the form of expectation-based noise reduction used in these simulations improves the ability of several different cognitive architectures to simulate human performance is quite compelling. Noise reduction via contingent processing appears to be an extremely efficient means of processing speech, even for architectures that use this "noise" to categorize (i.e. exemplar models).

New models of speech perception

Although our initial goal was to investigate how informational assumptions impact categorization systems, this study also involved the development of two novel models of speech perception. Although there have been discussions of the use of exemplar architectures in speech (e.g., Creel et al., 2008; Nygaard \& Pisoni, 1998), and computational models illustrating the principles of exemplar processing (Goldinger, 1998), no formal exemplar model had been developed using actual speech data for categorization. Similarly, PDP models and their mechanisms have been invoked in discussions of speech perception and word recognition (e.g., Elman \& Zipser, 1988; McClelland \& Elman, 1986); however, a full-scale model capable of learning how to weight and use cue values had not, to our knowledge, been proposed.

Our implementation of these models has demonstrated that both architectures are viable ways to simulate speech perception (although neither architecture was intended as a full simulation of speech perception). The logistic regression model proposed by McMurray and Jongman (2011), which operates similarly to other prototype-like models (e.g. NAPP: Nearey, 1990; FLMP: Oden \& Massaro, 1978) is capable of impressive performance with speech stimuli. However, both models presented herein offer comparable, and occasionally better, performance than logistic regression. Given more complex or unbalanced data-sets these architectures may be quite a bit better. This serves as an existence proof that such architectures are capable of supporting humanlike speech perception using cue values derived from actual speech tokens.

Our simulations also have implications for future work on these architectures in speech perception. First, in exemplar models, the $\tau$ and $r$ parameters on the similarity metric, the number of comparison tokens and the inclusion of other exemplars from similar talkers have major effects on the model's performance. Additionally, forcing the models to generalize by excluding matching talkers from the comparison set led to qualitatively different patterns of categorization. Future research should manipulate these factors to determine whether listeners perform fundamentally differently when performing categorization using known vs. unknown talkers.

For PDP models, we found, quite surprisingly, that increasing the number of hidden units did not allow raw models to accommodate acoustic variability. These models are expected to have the ability to embrace both statistical regularities and surface form information during learning. However, with increasing hidden units, the benefit of relative cues becomes progressively better. Although having a larger number of hidden units allows the network to encode additional patterns in the stimuli, these networks do not appear to use this information to harness context-specific information for categorization, perhaps because they do not provide adequate pressure for the model to form abstract stimulus representations.

\section{Mechanisms of relativization?}

Our simulations provide strong support for a speech perception system that incorporates expectations during perceptual encoding. However, our models do not explicitly simulate this recoding, but instead approximate it by passing the acoustic measurements through a linear regression before they enter the categorization model. This simplification allowed us to compare models with identical structures and allowed more straightforward analysis of architectures. We did not set out to 
simulate the entire process of learning and processing phonetic information, but instead to show how different forms of the input to a categorization system affect performance. However, this simplifying assumption is a far cry from an actual mechanism of relative encoding. Here we consider three aspects of relative encoding that are necessary to address in order to develop a mechanism: (1) At what level does relativization occur? (2) When during online processing does it occur? (3) What is actually occurring when this relativization occurs?

There are multiple possible levels at which relativization could occur. First, it may occur at the level of decisions (Smits, 2001a, b). Under this account, perceptual encoding is not affected by expectations, but instead expectations are used to moderate categorization decisions, especially in cases of perceptual ambiguity. In such an account, when a listener identifies the talker as a female, he can then use this information to alter decisions based on how he knows females produce individual cues; decisions for each cue become contingent on knowledge about talker (or other context) characteristics. This form of decision-level encoding is implemented by Smits's (2001b) HICAT model, as well as by ART (Grossberg \& Myers, 2000), and it is also quite comparable to how it might be implemented in loosely Bayesian approaches categorization. Under this view there is no adjustment of the sensory signal, but context does bias decisions.

Alternatively, relativization may occur at the level of perceptual encoding. Under such an account, the bottom-up information is affected by expectations or context which necessitates top-down expectations feeding back to alter perceptual encoding (e.g., Cole et al., 2010; McMurray \& Jongman, 2011). Knowledge about how talkers (or other contextual factors) affect production operates akin to a type of subtraction: the listener knows what the average male F0 is, so they can consider F0 relative to this value. ${ }^{11}$ Such an account is particularly appealing as it does not require adjusting a range of different decisions, but instead operates akin to regressing out sources of variance to account for all information in the signal (Fowler, 1986).

Cue-level relativization may offer a significant computational advantage over decision-level relativization. Consider the case of F0, a secondary cue for voicing. In a cue-level account, the listener can improve the value of this cue by recoding relative to the talker's gender - here, knowing that an F0 is high for a male is sufficient to improve the utility of this cue. In contrast, decision-level mechanisms require that the listener knows not only how F0 is impacted by gender, but also how this in turn should be used in making decisions about

\footnotetext{
${ }^{11}$ Note that this is quite different than the traditional conception of feedback in speech perception as an additive process (e.g., speech cues or phonemes are heard as more consistent with the context). Thus, this idea is quite distinct from the long-running debates over feedback in speech perception (e.g., McClelland, Mirman, \& Holt, 2006; Norris, McQueen, \& Cutler, 2000).
}

voicing. While if voicing was the only concern, these would appear to be fairly trivial differences. However, F0 can also be used as a cue to vowel identity and nasalization. Consequently, decision-level relativization may pose difficulties for generalizing knowledge of a contextual factor to the range of other processes it may play a role in Knowing how gender and F0 contribute to voicing does not help when F0 now has to be used for vowel identity. In contrast, by recoding at the cue-level, the relativized F0 can now be used for any judgment. That is, the relevant "knowledge" is about how gender and F0 interact in general, not how gender and F0 interact for categorizing particular properties of the signal. Thus, a relative encoding account that works by recoding the incoming sensory signal relative to expectations (much as in predictive coding schemes) may be much more flexible than one that merely incorporates context into decision making. Additionally, relativization need not occur at a single level of processing; instead, many accounts argue for pervasive relativization or expectation-based recoding across processing that (Carandini \& Heeger, 2011; Clark, 2012, McMurray et al., 2015).

We must also ask when relativization occurs during online processing. Speech unfolds over time; as information becomes available, listeners could immediately integrate all possible information sources, or they could wait until all relevant cues are gathered and then combine them. This latter approach suggests some form of buffer; in order to base a cue encoding on other information, the listener holds off making decisions until this later information is available. In many cases, such a buffer seems appealing: in our current dataset, the vowel (and therefore talker) information is not available until after the fricative, so a listener could wait for this later information to make a phonetic decision.

However, such a buffer may be inconsistent with empirical data showing immediate information integration. Although cues often arrive asynchronously, listeners appear to begin using each cue as soon as it is available (McMurray et al., 2008; Miller \& Dexter, 1988; Reinisch \& Sjerps, 2013; Toscano \& McMurray, 2012). Listeners thus make decisions based on whatever information is available at the moment, and can update these decisions to reflect later-occurring information. Immediate integration may be important in cases where it is unclear when all relevant information is available. In the case of fricatives, this may require listeners to use raw cues at early points in processing, and update their decisions as context becomes available for relativization. Indeed, our data show evidence that continuous updating must occur; information about talker and vowel are not available until after the fricative has been heard. This form of immediate activation with continued updating is similar to the resonances suggested in ARTPhone (Grossberg, 2003) and ARTWord (Grossberg \& Myers, 2000) models of Grossberg and colleagues. In these models, a "resonance" is formed as the listener integrates 
information across time. The initial information (in the case of the data used for the present models, the information during the fricative) starts a resonance that is updated as later information (the vowel, which likely also signals talker identity) arrives. This allows mutual information flow, both forward and backward, between different segments of the speech signal until a stable interpretation of the signal is reached.

The empirical support for this immediate integration has been conducted on stop consonant voicing, manner of articulation and vowels, and it is an open question whether it will extend to fricatives (though preliminary work suggests that listeners may delay processing of fricatives until later context is heard; Galle, 2014). However, McMurray and Jongman (2011) offer hints as to how it might work. They showed that a model based on raw cues does a good job of accounting for listener performance on the frication alone (when the vocoid, which contains the information about the talker and vowel, is excised form the segments), and the ER model was too accurate. Thus, listeners could engage in more continuous processing by using raw cues immediately and then integrating relativizing cues as they become available.

Finally, the close alignment of our ER encoding with predictive coding schemes also raises the question of the nature of prediction. Predictive coding is often posited as an anticipatory, forward-in-time, operation. For example, speakers may predict the sensory consequences of their own voice (Houde, Nagarajan, Sekihara, \& Merzenich, 2002), or the temporal structure of a word may predict future phonetic material (Gagnepain et al., 2012). This is clearly true in our case-knowing the talker predicts a particular form of the fricative. However, the particular framework in which we are working also suggests a role for retrospective reanalysis. That is, the relevant context may come after the target sound, requiring the target sound to be reanalyzed as a form of postdiction.

Indeed, a recent study using cross-spliced fricative-vowel syllables showed that listeners are less accurate at identifying fricatives when later-occurring vowel and/or talker information is misleading, lending support to immediate integration of information as it arrives for fricatives, even when this information occurs after the target speech segment (Apfelbaum et al., 2014). While this type of coding is not often discussed in predictive coding schemes more generally, it is not inconsistent with them. However, it may be much more important in the context of temporally unfolding speech, than in motor control (where prospective control is more important) or vision, the typical domains in which predictive coding is discussed.

Putting these ideas together, we suggest that the relativization described in our study may result from some form of feedback connections that alter perceptual information to reflect new information sources. In addition to predictive expectations from previous context, these feedback connections can augment ongoing processing to reflect later information, in a form of postdiction (Apfelbaum et al., 2014). This account can allow ready integration of information whenever it becomes available, it can accommodate information from multiple modalities, and it accords with current research on realtime speech perception. Additionally, a feedback-based account of information integration in speech perception fits well with effects of information from other levels of language processing affecting speech perception such as lexical or sentential content (Connine, Blasko, \& Hall, 1991; Ganong, 1980). However, it is also possible that relativization occurs several times in processing, and operates in different ways (Carandini \& Heeger, 2011). Ultimately, some form of realtime interactive activation operating at the interface between cues and categories may be a viable account of this.

\section{Theories of speech perception}

The finding that speech perception appears to rely on perceptual encoding mechanisms that are sensitive to expectations has major implications for theories of speech. Speech perception research has long focused on the problem of lack of invariance: the same speech sound is produced differently depending on factors like talker, phonological context and speaking rate. Theories that integrate some combination of the signal and cognitive expectations have proven quite successful at explaining speech categorization. Specifically, both C-CuRE (Cole et al., 2010; McMurray et al., 2011; McMurray \& Jongman, 2011) and the closely related HICAT (Smits, 2001a, b) offer close computational fits to human perceptual data and have made successful predictions of human performance. These theories argue that listeners first make a decision about an aspect of the acoustic signal or context (e.g. identifying the talker or a phoneme), and then use this information to bias encoding of other portions of the signal. Thus in this account, information is encoded in a relative fashion.

While these theories focus on cognitive expectations, other forms of relative encoding have been posited at other levels of the system. In auditory contrast accounts, listeners can adjust the encoding of a cue based on how that cue has behaved in surrounding acoustic environments (Coady, Kluender, \& Rhode, 2003; Holt, 2006; Kiefte \& Kluender, 2008; Kluender, Coady, \& Kiefte, 2003; Lotto \& Kluender, 1998; though see Viswanathan, Fowler, \& Magnuson, 2009). Such local-context contrast effects emerge even when the surrounding acoustic context does not include speech information (Holt, 2006; Lotto \& Kluender, 1998) and when the categorizer is a bird (Lotto, Kluender, \& Holt, 1997), suggesting that contrast effects are a general effect of the auditory processing system.

While these are broadly consistent with our relative coding account, auditory contrast alone may not account for relative processing in speech perception. First, contrast effects occur 
within a class of similar cues; for example, the F3 value from preceding context affects the way a listener uses F3 to identify place of articulation of a consonant in a contrastive manner (Lotto \& Kluender, 1998), and the value of F2 in a vowel can be relativized against the F1 value (Ladefoged \& Broadbent, 1957). However, relative encoding effects are often suggested to involve changes that cross types of cues, because this relativization is mediated by higher level expectations. For example, McMurray and Jongman (2011) suggest that cues to vowel identity can affect encoding of entirely different cues in a preceding fricative, such as fricative duration (see also, Apfelbaum et al., 2014). It is unclear how a pure contrast account could accommodate cross-cue effects (see also Toscano \& McMurray, 2014).

Second, the information listeners use to relativize perceptual encoding is not even always acoustic. Listeners identifying sounds along a continuum from $/ \mathrm{s} /$ to $/ \mathrm{J} /$ are influenced by knowing the gender of the talker, even if they learn this gender by seeing a male or a female face (Johnson et al., 1999; Strand, 1999; see also, Carden et al., 1981; Drager, 2011; Hay \& Drager, 2010; Magnuson \& Nusbaum, 2007; Niedzielski, 1999, for related demonstrations). Through previous experience, listeners come to know that females tend to produce these fricatives with a higher pitch than males, so they can use this knowledge to color their encoding of the tokens. The auditory contrast account argues that relative encoding is relative only to neighboring acoustic information; social factors affecting encoding are thus incompatible with this account.

This does not suggest that auditory contrast does not play a role in speech perception; the various studies from Kluender, Lotto, Holt and colleagues offer compelling evidence for such a mechanism (though see, Viswanathan et al., 2009). Rather, it is likely that some form of relativization occurs at several points during processing (Carandini \& Heeger, 2011, McMurray et al., 2015). Moreover, both forms of relativization are quite similar at a basic level: both operate to maximize contrast, either between cues, or between cues and expectations.

Finally, other non-veridical speech perception systems have been proposed. The motor theory (Liberman \& Mattingly, 1985) and direct realism (Fowler, 1986) argue that listeners extract some underlying form of the of the articulation or gestural intentions of the talker rather than using veridical encoding. Although such theories are suggested at a very different level of analysis, as they are not formally implementable, they may rely on quite similar mechanisms to those suggested here. Indeed the relativization mechanism proposed by C-CuRE is conceptually quite similar to gestural parsing (Fowler \& Smith, 1986; Fowler \& Brown, 2000), though it is also broader in scope (see, McMurray \& FarrisTrimble, 2012). These approaches argue that rather than independently making judgments about different speech segments, listeners attempt to identify all parts of the signal simultaneously, and that these judgments constrain each other. This results in something like relativization. For example, when listeners hear the word can, once they identify the final consonant as a nasal, they can attribute any nasality in the vowel to coarticulation, and hear the vowel as more oral (Fowler \& Brown, 2000). Thus the mechanisms entailed in motor or gestural accounts of speech perception are quite amenable to the form of relativization proposed here, and our simultaneous parsing across many acoustic cues may serve functionally the same purpose. However, as currently described, gestural theories have been applied only to the problem of compensating for coarticulation, not to factors like talker. As a result, these specific mechanisms are incapable of accommodating expectations derived from non-auditory sources such as social or talker information. In contrast ER models like C-CuRE can do this using the same mechanism (McMurray \& Farris-Trimble, 2012).

Similarly, adaptive resonance theory incorporates mechanisms that can accommodate a form of relative encoding in speech (Grossberg \& Myers, 2000; Grossberg, 2003). By merging bottom-up and top-down information to form a resonant representation of incoming information, these models adjust perception with respect to surrounding context. Although these models emphasize top-down information based on auditory contrast or neighboring phonetic decisions, there is no reason such a model could not incorporate other sources of information in the top-down information sources; social information, such as talker identity or expectations of dialect, could play into the way the resonances form, quite akin to the ER encoding scheme suggested by C-CuRE.

\section{Categorization more broadly}

Our results also bear on theories of categorization more broadly. Previous work has often remained agnostic as to information encoding prior to categorization; researchers focused on differentiating models that make extremely similar predictions, and so used stimuli that allowed precise control and clear category definitions (e.g., Smith, 2002). In cases when such categories were not an option, some researchers found they could still ignore encoding processes by focusing on the statistical relationships between stimuli rather than actual representations of these stimuli (Soto \& Wasserman, 2010). However, categorization in natural domains, such as face perception, may also confront issues of variability that are comparable to those in speech (Riesenhuber \& Poggio, 2000).

Fuller understanding of categorization, especially in the context of more natural stimuli that are more difficult to define in terms of clear-cut dimensions, may thus require a better appreciation of how perceptual information is initially encoded. Our models focused on speech information, which is notoriously multidimensional (Hillenbrand et al., 1995; 
Jongman et al., 2000; McMurray \& Jongman, 2011), whose categories often overlap quite significantly (Hillenbrand et al., 1995; Miller et al., 1986), and where cues are multiply determined by multiple classes of categories (e.g., talker and phoneme). These qualities of speech have led information content to the forefront of discussions of speech perception. Taking an approach to speech perception that puts all the work on categorization may thus lead us to overlook crucial parts of the categorization process.

Instead, we advocate investigating categorization architecture and perceptual encoding simultaneously to arrive at a fuller picture of categorization in this natural domain. Indeed, considering these simulations along with the prototype models of McMurray and Jongman (2011), we see that information content plays a massive role in model performance, perhaps even larger than the role of architecture itself. All three classes of models exhibited close matches to human performance, but in every case, models using ER cues performed better. This suggests a central role for relative cue encoding in speech perception across different theories of categorization.

This consideration of information content may bear on other natural categorization tasks. Multiple information sources often impinge on decision processes, forcing the categorizer to account for complex sources of variability (Reynolds \& Heeger, 2009). For example, face recognition relies on a confluence of factors that influence the perceptual form of a face. Many of these factors are interrelated and difficult to discriminate (e.g., Goldstone et al., 2003). Models of face categorization may benefit from detailed consideration of the processes underlying perceptual encoding of facial features; for example, coding a feature like eyebrow height relative to expectations for a given gender may help decode the identity or emotional expression of the face. Similarly, investigations of color categorization may demand that we simultaneously consider how viewers compensate for ambient light and shading, and the use of color as a cue for higher level categories may further drive a need for this. Although traditional categorization approaches, which are agnostic as to exact encoding processes, can explain some aspects of face and color categorization, this and other domains involving highly complex categorization tasks likely are more thoroughly explored using investigations of both architecture and information.

\footnotetext{
Acknowledgments This research was supported by National Institutes of Health Grant DC-008089 to B.M. and the Ballard and Seashore Dissertation Year Fellowship to K.A. We thank Yue Wang and Dan McEchron for help collecting and analyzing the acoustic data used in the simulations, and especially Jennifer Cole and Allard Jongman for invaluable help discussing theoretical components of this work. We also thank Robert Nosofsky and David Plaut for comments on a previous version of this manuscript.
}

\section{References}

Apfelbaum, K. S., Bullock-Rest, N., Rhone, A. E., Jongman, A., \& McMurray, B. (2014). Contingent categorization in speech perception. Language, Cognition and Neuroscience, 29(9), 1070-1082. doi:10.1080/01690965.2013.824995

Apfelbaum, K. S., \& McMurray, B. (2011). Using variability to guide dimensional weighting: Associative mechanisms in early word learning. Cognitive Science, 35(6), 1105-1138. doi:10.1111/j. 1551-6709.2011.01181.x

Blumstein, S. E., \& Stevens, K. N. (1979). Acoustic invariance in speech production: Evidence from measurements of the spectral characteristics of stop consonants. Journal of the Acoustical Society of America, 66(4), 1001-1017.

Blumstein, S. E., \& Stevens, K. N. (1980). Perceptual invariance and onset spectra for stop consonants in different vowel environments. The Journal of the Acoustical Society of America, 67(2), 648-662.

Bradlow, A. R., Nygaard, L. C., \& Pisoni, D. B. (1999). Effects of talker, rate, and amplitude variation on recognition memory for spoken words. Perception and Psychophysics, 61(2), 206-219.

Carandini, M., \& Heeger, D. J. (2011). Normalization as a canonical neural computation. Nature Reviews. Neuroscience, 13, 51-62. doi: 10.1038/nrn3136

Carden, G., Levitt, A., Jusczyk, P. W., \& Walley, A. C. (1981). Evidence for phonetic processing of cues to place of articulation: Perceived manner affects perceived place. Perception and Psychophysics, 29(1), 26-36.

Carney, A. E., Widin, G. P., \& Viemeister, N. F. (1977). Noncategorical perception of stop consonants differing in VOT. The Journal of the Acoustical Society of America, 62(4), 961-970. doi:10.1121/1. 381590

Clark, A. (2012). Whatever next? Predictive brains, situated agents, and the future of cognitive science. Behavioral and Brain Sciences, 186.

Clark, A. (2013). Expecting the World: Perception, Prediction, and the Origins of Human Knowledge. Journal of Philosophy, CX(9), 469-496.

Coady, J. A., Kluender, K. R., \& Rhode, W. S. (2003). Effects of contrast between onsets of speech and other complex spectra. The Journal of the Acoustical Society of America, 114(4), 2225-2235. doi:10.1121/ 1.1608955

Cole, J. S., Linebaugh, G., Munson, C., \& McMurray, B. (2010). Unmasking the acoustic effects of vowel-to-vowel coarticulation: A statistical modeling approach. Journal of Phonetics, 38(2), 167184. doi:10.1016/j.wocn.2009.08.004

Connine, C. M., Blasko, D. G., \& Hall, M. (1991). Effects of subsequent sentence context in auditory word recognition: Temporal and linguistic constrainst. Journal of Memory and Language, 30(2), 234 250. doi:10.1016/0749-596X(91)90005-5

Creel, S. C., Aslin, R. N., \& Tanenhaus, M. K. (2008). Heeding the voice of experience: The role of talker variation in lexical access. Cognition, 106(2), 633-664. doi:10.1016/j.cognition.2007.03.013

Drager, K. (2011). Speaker age and vowel perception. Language and Speech, 54(1), 99-121. doi:10.1177/0023830910388017

Elman, J. L., \& Zipser, D. (1988). Learning the hidden structure of speech. The Journal of the Acoustical Society of America, 83(4), 1615-1626.

Fowler, C. A. (1986). An event approach to the study of speech perception from a direct-realist perspective. Journal of Phonetics, 14, 3-28.

Fowler, C. A., \& Brown, J. M. (2000). Perceptual parsing of acoustic consequences of velum lowering from information for vowels. Perception and Psychophysics, 62(1), 21-32.

Fowler, C. A., \& Smith, M. (1986). Speech perception as "vector analysis": An approach to the problems of segmentation and invariance. In J. S. Perkell \& D. Klatt (Eds.), Invariance and Variability in Speech Processes (pp. 126-136). Hillsdale, NJ: Erlbaum. 
Frye, R. E., Fisher, J. M., Coty, A., Zarella, M., Liederman, J., \& Halgren, E. (2007). Linear coding of voice onset time. Journal of Cognitive Neuroscience, 19(9), 1476-1487. doi:10.1162/jocn.2007.19.9.1476

Gagnepain, P., Henson, R. N., \& Davis, M. H. (2012). Temporal predictive codes for spoken words in auditory cortex. Current Biology, 22(7), 615-621. doi:10.1016/j.cub.2012.02.015

Galle, M. (2014). Integration of asynchronous cues in fricative perception in real-time and developmental-time: Evidence for sublexical memory/integration systems. (PhD), University of Iowa.

Ganong, W. F. (1980). Phonetic categorization in auditory word perception. Journal of Experimental Psychology: Human Perception and Performance, 6(1), 110-125.

Gerrits, E., \& Schouten, M. E. H. (2004). Categorical perception depends on the discrimination task. Perception and Psychophysics, 66(3), 363-376.

Goldinger, S. D. (1998). Echoes of echoes? An episodic theory of lexical access. Psychological Review, 105(2), 251-279.

Goldstone, R. L., Steyvers, M., \& Rogosky, B. J. (2003). Conceptual interrelatedness and caricatures. Memory \& Cognition, 31(2), 169-180.

Grossberg, S. (2003). Resonant neural dynamics of speech perception. Journal of Phonetics, 31(3-4), 423-445. doi:10.1016/S00954470(03)00051-2

Grossberg, S., \& Myers, C. (2000). The resonant dynamics of speech perception: Interword integration and duration-dependent backward effects. Psychological Review, 107(4), 735-767.

Hawkins, S. (2003). Roles and representations of systematic fine phonetic detail in speech understanding. Journal of Phonetics, 31(3-4), 373405. doi:10.1016/j.wocn.2003.09.006

Hay, J., \& Drager, K. (2010). Stuffed toys and speech perception. Linguistics, 4(2010), 865-892. doi:10.1515/LING.2010.027

Hillenbrand, J. M., Clark, M. J., \& Nearey, T. M. (2001). Effects of consonant environment on vowel formant patterns. The Journal of the Acoustical Society of America, 109(2), 748-763. doi:10.1121/1.1337959

Hillenbrand, J. M., Getty, L. A., Clark, M. J., \& Wheeler, K. (1995). Acoustic characteristics of American English vowels. The Journal of the Acoustical Society of America, 97(5), 3099-3111. doi:10. $1121 / 1.411872$

Holt, L. L. (2006). The mean matters: Effects of statistically defined nonspeech spectral distributions on speech categorization. The Journal of the Acoustical Society of America, 120(5), 2801-2817. doi:10.1121/1.2354071

Hornik, K., Stinchcombe, M., \& White, H. (1989). Multilayer feedforward networks are universal approximators. Neural Networks, 2, 359-366.

Houde, J. F., Nagarajan, S. S., Sekihara, K., \& Merzenich, M. M. (2002). Modulation of the auditory cortex during speech: An MEG study. Journal of Cognitive Neuroscience, 14(8), 1125-1138. doi:10.1162/ 089892902760807140

Johnson, K., Strand, E. A., \& D'Imperio, M. (1999). Auditory-visual integration of talker gender in vowel perception. Journal of Phonetics, 27(4), 359-384. doi:10.1006/jpho.1999.0100

Jongman, A., Wayland, R., \& Wong, S. (2000). Acoustic characteristics of English fricatives. The Journal of the Acoustical Society of America, 108(3 Pt 1), 1252-1263.

Juneja, A., \& Espy-Wilson, C. (2008). A probabilistic framework for landmark detection based on phonetic features for automatic speech recognition. The Journal of the Acoustical Society of America, 123(2), 1154-1168. doi:10.1121/1.2823754

Kiefte, M., \& Kluender, K. R. (2008). Absorption of reliable spectral characteristics in auditory perception. The Journal of the Acoustical Society of America, 123(1), 366-376. doi:10.1121/1.2804951

Kluender, K. R., Coady, J. A., \& Kiefte, M. (2003). Sensitivity to change in perception of speech. Speech Communication, 41(1), 59-69. doi: 10.1016/S0167-6393(02)00093-6

Kruschke, J. K. (1992). ALCOVE: An exemplar-based connectionist model of category learning. Psychological Review, 99(1), 22-44.
Ladefoged, P., \& Broadbent, D. (1957). Information conveyed by vowels. The Journal of the Acoustical Society of America, 29(1), 98-104.

Lahiri, A., Gewirth, L., \& Blumstein, S. E. (1984). A reconsideration of acoustic invariance for place of articulation in diffuse stop consonants: Evidence from a cross-language study. The Journal of the Acoustical Society of America, 76(2), 391-404.

Liberman, A. M., \& Cooper, F. (1967). Perception of the speech code. Psychological Review, 74(6), 431-461.

Liberman, A. M., Harris, K. S., Hoffman, H. S., \& Griffith, B. C. (1957). The discrimination of speech sounds within and across phoneme boundaries. Journal of Experimental Psychology, 54(5), 358-368.

Liberman, A. M., \& Mattingly, I. G. (1985). The motor theory of speech perception revised. Cognition, 21(1), 1-36.

Lindblom, B. (1996). Role of articulation in speech perception: Clues from production. The Journal of the Acoustical Society of America, 99(3), 1683-1692. doi:10.1121/1.414691

Lisker, L. (1986). "Voicing" in English: A catalogue of acoustic features signaling /b/ versus /p/ in trochees. Language and Speech, 29, 3-11. doi:10.1177/002383098602900102

Lotto, A. J., \& Kluender, K. R. (1998). General contrast effects in speech perception: Effect of preceding liquid on stop consonant identification. Perception and Psychophysics, 60(4), 602-619.

Lotto, A. J., Kluender, K. R., \& Holt, L. L. (1997). Perceptual compensation for coarticulation by Japanese quail (Coturnix coturnix japonica). The Journal of the Acoustical Society of America, 102(2), 1134-1140. doi:10.1121/1.419865

Love, B. C., Medin, D. L., \& Gureckis, T. M. (2004). SUSTAIN: A network model of category learning. Psychological Review, 111(2), 309-332. doi:10.1037/0033-295X.111.2.309

Maddox, W. T., Bohil, C. J., \& Ing, A. D. (2004). Evidence for a procedural-learning-based system in perceptual category learning. Psychonomic Bulletin \& Review, 11(5), 945-952.

Magnuson, J. S., \& Nusbaum, H. C. (2007). Acoustic differences, listener expectations, and the perceptual accommodation of talker variability. Journal of Experimental Psychology: Human Perception and Performance, 33(2), 391-409. doi:10.1037/0096-1523.33.2.391

Massaro, D. W., \& Cohen, M. (1983). Categorical or continuous speech perception: A new test. Speech Communication, 2, 15-35.

McClelland, J. L., \& Elman, J. L. (1986). The TRACE model of speech perception. Cognitive Psychology, 18(1), 1-86.

McClelland, J. L., Mirman, D., \& Holt, L. L. (2006). Are there interactive processes in speech perception? Trends in Cognitive Sciences, 10(8), 363-369. doi:10.1016/j.tics.2006.06.007

McLennan, C. T., \& Luce, P. A. (2005). Examining the time course of indexical specificity effects in spoken word recognition. Journal of Experimental Psychology: Learning, Memory, and Cognition, 31(2), 306-321. doi:10.1037/0278-7393.31.2.306

McMurray, B., Clayards, M. A., Tanenhaus, M. K., \& Aslin, R. N. (2008). Tracking the time course of phonetic cue integration during spoken word recognition. Psychonomic Bulletin \& Review, 15(6), 1064-1071. doi:10.3758/PBR.15.6.1064

McMurray, B., Cole, J. S., \& Munson, C. (2011). Features as an emergent product of computing perceptual cues relative to expectations. In R. Ridouane \& N. Clement (Eds.), Where Do Features Come From? (pp. 197-236). Amsterdam: Benjamins.

McMurray, B., \& Farris-Trimble, A. (2012). Emergent information-level coupling between perception and production. In A. Cohn, C. Fougeron, \& M. Huffman (Eds.), The Oxford Handbook of Laboratory Phonology. Oxford: Oxford University Press.

McMurray, B., \& Jongman, A. (2011). What information is necessary for speech categorization? Harnessing variability in the speech signal by integrating cues computed relative to expectations. Psychological Review, 118(2), 219-246. doi:10. 1037/a0022325

McMurray, B., Rhone, A. E., \& Hannaway, K. (2015). Relativity in speech perception: From locus equations to predictive coding. In 
A. Agwuele \& A. Lotto (Eds.), Essays in speech processes: Language production and perception. Equinox: Sheffield, UK.

Medin, D. L., \& Schaffer, M. M. (1978). Context theory of classification learning. Psychological Review, 85(3), 207-238.

Mermelstein, P. (1978). On the relationship between vowel and consonant identification when cued by the same acoustic information. Perception \& Psychophysics, 23(4), 331-336.

Miller, J. L., \& Dexter, E. R. (1988). Effects of speaking rate and lexical status on phonetic perception. Journal of Experimental Psychology: Human Perception and Performance, 14(3), 369-378.

Miller, J. L., Green, K. P., \& Reeves, A. (1986). Speaking rate and segments: A look a the relation between speech production and speech perception for the voicing contrast. Phonetica, 43, 106-115.

Minda, J. P., \& Smith, J. D. (2002). Comparing prototype-based and exemplar-based accounts of category learning and attentional allocation. Journal of Experimental Psychology: Learning, Memory, and Cognition, 28(2), 275-292.

Myers, E. B., Blumstein, S. E., Walsh, E., \& Eliassen, J. (2009). Inferior frontal regions underlie the perception of phonetic category invariance. Psychological Science, 20(7), 895-903. doi:10.1111/j.14679280.2009.02380.x

Nearey, T. M. (1989). Static, dynamic, and relational properties in vowel perception. The Journal of the Acoustical Society of America, 85(5), 2088-2113. doi:10.1121/1.397861

Nearey, T. M. (1990). The segment as a unit of speech perception. Journal of Phonetics, 18, 347-373.

Nearey, T. M. (1997). Speech perception as pattern recognition. The Journal of the Acoustical Society of America, 101(6), 3241-3254.

Nearey, T. M., \& Hogan, J. T. (1986). Phonological contrast in experimental phonetics: Relating distributions of production data to perceptual categorization curves. Experimental Phonology, 141-161.

Niedzielski, N. (1999). The effect of social information on the perception of sociolinguistic variables. Journal of Language and Social Psychology, 18(1), 62-85. doi:10.1177/0261927X99018001005

Norris, D., McQueen, J. M., \& Cutler, A. (2000). Merging information in speech recognition: Feedback is never necessary. Behavioral and Brain Sciences, 23(3).

Nosofsky, R. M. (1986). Attention, similarity, and the identificationcategorization relationship. Journal of Experimental Psychology: General, 115(1), 39-61.

Nosofsky, R. M. (1988). Similarity, frequency, and category representations. Journal of Experimental Psychology: Learning, Memory, and Cognition, 14(1), 54-65.

Nosofsky, R. M., Kruschke, J. K., \& McKinley, S. C. (1992). Combining exemplar-based category representations and connectionist learning rules. Journal of Experimental Psychology: Learning, Memory, and Cognition, 18(2), 211-233.

Nygaard, L. C., \& Pisoni, D. B. (1998). Talker-specific learning in speech perception. Perception and Psychophysics, 60(3), 355-376.

Nygaard, L. C., Sommers, M. S., \& Pisoni, D. B. (1994). Speech perception as a talker-contingent process. Psychological Science, 5(1), 42-46. doi:10.1111/j.1467-9280.1994.tb00612.x

Oden, G. C., \& Massaro, D. W. (1978). Integration of featural information in speech perception. Psychological Review, 85(3), 172-191.

Ohala, J. J. (1996). Speech perception is hearing sounds, not tongues. The Journal of the Acoustical Society of America, 99(3), 1718-1725.

Pickering, M. J., \& Garrod, S. (2004). Toward a mechanistic psychology of dialogue. Behavioral and Brain Sciences, 27(2), 169-190. discussion 190-226.

Pierrehumbert, J. B. (2003). Phonetic diversity, statistical learning, and acquisition of phonology. Language and Speech, 46(2-3), 115-154. doi:10.1177/00238309030460020501

Pind, J. (1995). Speaking rate, voice-onset time, and quantity: The search for higher-order invariants for two Icelandic speech cues. Attention, Perception, \& Psychophysics, 57(3), 291-304.
Pisoni, D. B., \& Tash, J. (1974). Reaction times to comparisons within and across phonetic categories. Perception and Psychophysics, 15(2), 285-290.

Pitt, M. A., Kim, W., Navarro, D. J., \& Myung, J. I. (2006). Global model analysis by parameter space partitioning. Psychological Review, 113(1), 57-83. doi:10.1037/0033-295X.113.1.57

Port, R. F., \& Dalby, J. (1982). Consonant/vowel ratio as a cue for voicing in English. Perception and Psychophysics, 32(2), 141-152.

Posner, M. I., \& Keele, S. W. (1968). On the genesis of abstract ideas. Journal of Experimental Psychology, 77(3), 353-363.

Reinisch, E., \& Sjerps, M. J. (2013). The uptake of spectral and temporal cues in vowel perception is rapidly influenced by context. Journal of Phonetics, 41(2), 101-116. doi:10.1016/j.wocn.2013.01.002

Reynolds, J. H., \& Heeger, D. J. (2009). The Normalization Model of Attention. Neuron, 61(2), 168-185. doi:10.1016/j.neuron.2009.01. 002

Rhone, A. E., \& Jongman, A. (2012). Modified locus equations categorize stop place in a perceptually realistic time frame. The Journal of the Acoustical Society of America, 131(6), EL487-EL491. doi:10. $1121 / 1.4722169$

Riesenhuber, M., \& Poggio, T. (2000). Models of object recognition. Nature Neuroscience, 3(Suppl), 1199-1204. doi:10.1038/81479

Rumelhart, D. E., Hinton, G. E., \& Williams, R. J. (1986). Learning representations by back-propagating errors. Nature, 323, 533-536.

Schouten, B., Gerrits, E., \& van Hessen, A. (2003). The end of categorical perception as we know it. Speech Communication, 41(1), 71-80. doi:10.1016/S0167-6393(02)00094-8

Smith, J. D. (2002). Exemplar theory's predicted typicality gradient can be tested and disconfirmed. Psychological Science, 13(5), 437-442.

Smith, J. D., \& Minda, J. P. (1998). Prototypes in the mist: The early epochs of category learning. Journal of Experimental Psychology: Learning, Memory, and Cognition, 24(6), 1411-1436.

Smits, R. (2001a). Evidence for hierarchical categorization of coarticulated phonemes. Journal of Experimental Psychology: Human Perception and Performance, 27(5), 1145-1162.

Smits, R. (2001b). Hierarchical categorization of coarticulated phonemes: A theoretical analysis. Perception and Psychophysics, 63(7), 11091139.

Soto, F. A., \& Wasserman, E. A. (2010). Error-driven learning in visual categorization and object recognition: A common-elements model. Psychological Review, 117(2), 349-381. doi:10.1037/a0018695

Stevens, K. N. (2002). Toward a model for lexical access based on acoustic landmarks and distinctive features. The Journal of the Acoustical Society of America, 111(4), 1872-1891. doi:10.1121/1. 1458026

Stevens, K. N., \& Keyser, S. J. (2010). Quantal theory, enhancement and overlap. Journal of Phonetics, 38(1), 10-19. doi:10.1016/j.wocn. 2008.10.004

Strand, E. A. (1999). Uncovering the role of gender stereotypes in speech perception. Journal of Language and Social Psychology, 18(1), 86100. doi:10.1177/0261927X99018001006

Summerfield, Q. (1981). Articulatory rate and perceptual constancy in phonetic perception. Journal of Experimental Psychology: Human Perception and Performance, 7(5), 1074-1095.

Sussman, H. M., Fruchter, D., Hilbert, J., \& Sirosh, J. (1998). Linear correlates in the speech signal: The orderly output constraint. Behavioral and Brain Sciences, 21(02), 241-299. doi:10.1017/ S0140525X98001174

Toscano, J. C., \& McMurray, B. (2010). Cue integration with categories: Weighting acoustic cues in speech using unsupervised learning and distributional statistics. Cognitive Science, 34(3), 434-464. doi:10. 1111/j.1551-6709.2009.01077.x

Toscano, J. C., \& McMurray, B. (2012). Cue-integration and context effects in speech: Evidence against speaking-rate normalization. Attention, Perception, \& Psychophysics, 74(6), 1284-1301. doi: 10.3758/s13414-012-0306-Z 
Toscano, J. C., \& McMurray, B. (2014). The time-course of speaking rate compensation: Effects of sentential rate and vowel length on voicing judgments. Language, Cognition and Neuroscience. doi:10.1080/ 23273798.2014.946427

Toscano, J. C., McMurray, B., Dennhardt, J., \& Luck, S. J. (2010). Continuous perception and graded categorization: Electrophysiological evidence for a linear relationship between the acoustic signal and perceptual encoding of speech. Psychological Science, 21(10), 1532-1540. doi:10.1177/ 0956797610384142

Viswanathan, N., Fowler, C. A., \& Magnuson, J. S. (2009). A critical examination of the spectral contrast account of compensation for coarticulation. Psychonomic Bulletin \& Review, 16(1), 74-79. doi: 10.3758/PBR.16.1.74

Von Kriegstein, K., Smith, D. R. R., Patterson, R. D., Kiebel, S. J., \& Griffiths, T. D. (2010). How the human brain recognizes speech in the context of changing speakers. The Journal of Neuroscience, 30(2), 629-638. doi:10.1523/JNEUROSCI. 2742-09.2010

Whalen, D. H. (1989). Vowel and consonant judgments are not independent when cues by the same information. Attention, Perception, \& Psychophysics, 46(3), 284-292.

Whalen, D. H. (1992). Perception of overlapping segments: Thoughts on Nearey's model. Journal of Phonetics, 20, 493-496.

Yu, C., \& Smith, L. B. (2012). Modeling cross-situational word-referent learning: Prior questions. Psychological Review, 119(1), 21-39. doi: 10.1037/a0026182

Zacks, J. M., Speer, N. K., Swallow, K. M., Braver, T. S., \& Reynolds, J. R. (2007). Event perception: A mind-brain perspective. Psychological Bulletin, 133(2), 273-293. doi:10.1037/0033-2909.133.2.273

Zaki, S. R., \& Nosofsky, R. M. (2007). A high-distortion enhancement effect in the prototype-learning paradigm: Dramatic effects of category learning during test. Memory \& Cognition, 35(8), 2088-2096. 